\title{
Genoarchitectonic
}

\section{Compartmentalization of the} Embryonic Telencephalon: Insights From the Domestic Cat

\author{
Nikistratos Siskos ${ }^{1}$, Charalampos Ververidis ${ }^{2}$, George Skavdis ${ }^{3}$ and Maria E. Grigoriou ${ }^{1 *}$ \\ ${ }^{1}$ Laboratory of Developmental Biology \& Molecular Neurobiology, Department of Molecular Biology \& Genetics, Democritus \\ University of Thrace, Alexandroupolis, Greece, ${ }^{2}$ Obstetrics and Surgery Unit, Companion Animal Clinic, School of Veterinary \\ Medicine, Faculty of Health Sciences, Aristotle University of Thessaloniki, Thessaloniki, Greece, ${ }^{3}$ Laboratory of Molecular \\ Regulation \& Diagnostic Technology, Department of Molecular Biology \& Genetics, Democritus University of Thrace, \\ Alexandroupolis, Greece
}

OPEN ACCESS

Edited by:

José L. Ferran,

University of Murcia, Spain

Reviewed by:

Luis Puelles,

University of Murcia, Spain

Loreta Medina,

Universitat de Lleida, Spain

*Correspondence:

Maria E. Grigoriou

mgrigor@mbg.duth.gr

Received: 29 September 2021 Accepted: 16 November 2021 Published: 16 December 2021

Citation:

Siskos N, Ververidis C, Skavdis G and Grigoriou ME (2021)

Genoarchitectonic

Compartmentalization of the Embryonic Telencephalon: Insights

From the Domestic Cat.

Front. Neuroanat. 15:785541. doi: 10.3389/fnana.2021.785541
The telencephalon develops from the alar plate of the secondary prosencephalon and is subdivided into two distinct divisions, the pallium, which derives solely from prosomere hp1, and the subpallium which derives from both hp1 and hp2 prosomeres. In this first systematic analysis of the feline telencephalon genoarchitecture, we apply the prosomeric model to compare the expression of a battery of genes, including Tbr 1 , Tbr2, Pax6, Mash1, Dlx2, Nkx2-1, Lhx6, Lhx7, Lhx2, and Emx1, the orthologs of which alone or in combination, demarcate molecularly distinct territories in other species. We characterize, within the pallium and the subpallium, domains and subdomains topologically equivalent to those previously described in other vertebrate species and we show that the overall genoarchitectural map of the E26/27 feline brain is highly similar to that of the E13.5/E14 mouse. In addition, using the same approach at the earlier (E22/23 and E24/25) or later (E28/29 and E34/35) stages we further analyze neurogenesis, define the timing and duration of several developmental events, and compare our data with those from similar mouse studies; our results point to a complex pattern of heterochronies and show that, compared with the mouse, developmental events in the feline telencephalon span over extended periods suggesting that cats may provide a useful animal model to study brain patterning in ontogenesis and evolution.

Keywords: feline brain development, prosomeric model, genoarchitecture, embryonic telencephalon, patterning

Abbreviations: AA, Anterior Amygdala; AOB, Accessory olfactory bulb; AStr, Striatal amygdala; BAOT, Bed nucleus of the accessory olfactory tract; Ce, Central amygdalar nucleus; CGE, Caudal ganglionic eminence; Cl, Claustrum; CMS, Caudal migratory stream; cp, Cortical plate; CxH, Cortical hem; DB, Diagonal Band; Dg, Diagonal domain of the subpallium; DgSe, Septal subdivision of the Dg; DP, Dorsal Pallium; dS, Dorsal Striatal division; EA, Extended Amygdala; EPd, Dorsal Endopiriform Nucleus; GP, Globus Pallidus; Hi, Hippocampal anlage; hp1, Hypothalamic (or secondary prosencephalic) prosomere 1; hp2, Hypothalamic (or secondary prosencephalic) prosomere 2; ic, Internal capsule; ivf, Interventricular foramina; iz, Intermediate zone; LGE, Lateral Ganglionic Eminence; lot, Lateral olfactory tract; LP, Lateral Pallium; LPO, Lateral preoptic area; MCL, Mitral cell layer; MP, Medial Pallium; Acb, Nucleus Accumbens; NLOT, Nucleus of the lateral olfactory tract; OB, Olfactory bulb; ONL, Olfactory nerve layer; OT, Olfactory tubercule; Pal, Pallium; Pd, Pallidal domain; Pdm, Pallidal mantle; PdSe, Septal Pallidal subdivision; POA, Preoptic Area; POAm, Mantle of the POA; POASe, Septal subdivision of the POA; POH, Preoptic-hypothalamic border region; pp, Preplate; PSB, Pallial-subpallial boundary; PSe, Pallial Septum; PThE, Prethalamic Eminence; rf, Rhinal fissure; rld, Rostrolateral domain; SLEA, Sublenticular extended amygdala; SP, Subpallium; sp, Subplate; SPV, Supraoptic paraventricular region; Str, Striatal domain of the subpallium; StrSe, Septal striatal subdivision; svz, Subventricular zone; VAP, Ventropallial amygdalopiriform area; VAPvz, vz of the VAP; VAPm, Ventropallial amygdalopiriform area mantle; VP, Ventral Pallium; VPvz, vz of the VP; vS, Ventral Striatal division; vz, Ventricular zone. 


\section{INTRODUCTION}

Genoarchitectonics, by combining gene expression data with cell morphology and topology, has become in the past decade a powerful approach in the study of the nervous system (Puelles and Ferran, 2012). New ontogenetic construction of the brain of the mouse and other species have been described using genoarchitecture, leading to the revised prosomeric model and the relevant developmental ontology (Medina, 2007; Puelles et al., 2013; Watson et al., 2017). The telencephalon, the largest compartment of the mammalian central nervous system is a highly complex structure in terms of cytoarchitecture, hodology, and function; it derives from the alar plate of the secondary prosencephalon, a domain that corresponds to the anterior territory of the neural plate which, through the differential expression of several regulatory genes is subdivided into two morphological and molecular distinct divisions, namely, the pallium and the subpallium (Puelles et al., 2000; Puelles and Rubenstein, 2003, 2015; Watson et al., 2017). The telencephalon derives from prosomeres hp1 and hp2; the dorsal part of the former produces the evaginated alar plate and gives rise to the entire pallium and most of the subpallium, while the latter gives rise to the non-evaginated alar plate and produces the rest of the subpallium (Watson et al., 2017).

The pallium represents the anlagen of all the cortical areas (e.g., isocortex, allocortex, hippocampus, olfactory bulb) and pallial nuclear masses, such as the claustrum $(\mathrm{Cl})$ and the pallial amygdalar complex, and is molecularly identified by the expression of several genes, for instance, Emx1/2, Pax6, Lhx2/9, Tbr1/2, Nr4a2 (Nurr1), and Lef1. Within the pallium, four, radially arranged distinct territories have been defined by differential gene expression studies: (1) The ventral pallium (VP), the anlage of the olfactory bulb (OB), and the olfactory allocortex that expresses Pax6, Dbx1, Sfrp2, and Lhx9 (Puelles et al., 2000; Kim et al., 2001; Medina et al., 2004; García-López et al., 2008). (2) The lateral pallium (LP) is uniquely identified by claustral Nr4a2 expression and corresponds to the primordium of the claustroinsular complex (Puelles, 2014; Puelles et al., 2016a). (3) The dorsal pallium (DP), the isocortical anlage that expresses, among other genes, Lhx2, Emx1/2, and Pax6 (Puelles et al., 2000; Abellán et al., 2014). (4) The medial pallium (MP), the hippocampal primordium is indicatively identified by Lef1 and Lhx2/9 expression (Abellán et al., 2014). This tetrapartite model has been successfully applied to study pallial development in a wide range of vertebrates, including various tetrapod species (Medina et al., 2005; Puelles, 2017). Recent evidence in lacertids has proposed the existence of two additional domains, the dorsolateral and ventrocaudal pallia (Desfilis et al., 2018). Integrating these domains into the developmental ontology may upgrade our current understanding of the pallium as being hexapartite (Medina et al., 2019, 2021).

The subpallium, the primordium of the telencephalic basal ganglia contributes to the extended amygdala (EA), the medial, lateral, caudal ganglionic eminences (CGEs), the preoptic area, and the septum (Medina and Abellán, 2012). Moreover, it is the cradle of the cortical GABAergic interneurons (Lavdas et al., 1999). In contrast to the pallium, in the subpallium several genes, for instance, Gsx1/2, Dlx1/2, Mash1 (Ascl1), Nkx21 , and $L h x 6 / 7$, are expressed with a unique pattern (Flames et al., 2007). Genoarchitectonics divide the subpallium into four distinct histogenetic domains (Puelles et al., 2013): (1) The striatal (Str) division with major derivatives, the caudoputamen complex and the nucleus accumbens (Acb), molecularly defined by the presence of Gsx2, Dlx2, and Mash1, being devoid of Nkx2-1 expression. (2) The pallidal (Pd) domain, the anlage of the globus pallidus (GP) is mainly characterized by high Nkx2-1 along with Dlx2, Mash1, Gsx1, Lhx6 expression. (3) The preoptic area (POA), the anlage of the postnatal preoptic nuclei, that arises from hp2, corresponds to the non-evaginated telencephalon and uniquely expresses Shh. (4) The diagonal domain (Dg), interposed between the POA and the Pd, identified by the expression of Er81 in the $\mathrm{vz}$ which is devoid of Shh messenger RNA (mRNA); Dg is also associated with the specification of Somatostatin expressing neurons, and its derivatives include the postnatal nuclei of the diagonal band (DB) and the substantia innominata (Puelles et al., 2016b). Finally, in each histogenetic domain, four secondary subdivisions (i.e., the septal, the paraseptal, the central, and the amygdaloid subdivision) have been recognized along the septoamygdalar axis (Puelles et al., 2013).

The vast majority of the developmental genoarchitectonic studies have been carried out in mice, while data in other mammalian species are very scarce. The domestic cat (Felis catus) has been a popular animal model for biological research (Scott, 1977). In neurosciences in the second half of the 20th century, cats have been extensively used to study various aspects of the biology of the central nervous system (CNS), including the physiology of the $\mathrm{Cl}$ (for review please refer to Sherk, 2014) or the hodology of the amygdala (e.g., Krettek and Price, 1978a,b), while pioneering atlases were published (Jasper and AjmoneMarsan, 1954; Markowitsch and Pritzel, 1977). Of particular interest are considered works from the Shatz group that studied neurogenesis in the feline telencephalon (Luskin and Shatz, 1985b), including birth-dating the first neurons generated in the visual cortex (Luskin and Shatz, 1985a). In the 90s, however, the use of cat as a model organism declined (Manger et al., 2008); yet the sequencing of the feline genome revived the interest toward this species (Pontius et al., 2007; Buckley et al., 2020); this may also be attributed to the fact that it can be used as a model organism for several human diseases (e.g., FIV/FAIDS, FeCoV, or SARS-COV-2) and to develop and/or test novel molecular therapeutics (for instance see Bradbury et al., 2013; McCurdy et al., 2015; Gray-Edwards et al., 2017; Shi et al., 2020). In neurosciences, the domestic cat has been recently used in functional neuroimaging studies (Stolzberg et al., 2017), auditory or visual neurosciences (Butler et al., 2015; Kremkow et al., 2016; Galuske et al., 2019), neurophysiology (Darch et al., 2020), as well as neuroanatomy (Hinova-Palova et al., 2019). From a phylogenetic perspective, Laurasiatheria, the superorder from which felids sprouted, diverged before the separation of the human Euarchonta stem progenitor, from the murine Glires ancestor (Bininda-Emonds and Hartmann, 2017). The feline brain, in contrast with the lissencephalic mouse brain, is gyrencephalic (Zilles et al., 2013); this trait favors comparisons 
both with primates, as well as with the stem mammalian ancestor (Kelava et al., 2013; Catania, 2017). Additionally, felids can be regarded as an organism that provides a "bridge" between rodents and primates (Bradbury et al., 2013; Stolzberg et al., 2017); thus the study of the development of the feline brain not only can provide novel insights into the evolution of the mammalian brain but can also be useful for basic and translational neuroscience (e.g., in Graff et al., 2020).

In this work we have studied the genoarchitectonic organization of the embryonic feline telencephalon by analyzing the expression of several gene markers, the orthologs of which have been previously used in other species, to identify specific domains (e.g., Puelles et al., 2000; Flames et al., 2007; Abellán et al., 2014; Desfilis et al., 2018). We characterized, within the pallium and the subpallium, domains, and subdomains topologically equivalent to those that have been previously described in other vertebrate species and we studied the timing and duration of several developmental events. Our data show that the genoarchitectural map of the feline telencephalon is highly conserved. When compared with data from mouse studies heterochronies in the timing of developmental events were observed; moreover, developmental processes in the feline telencephalon span over extended periods suggesting that cats may provide a useful animal model for increasing our knowledge on the degree of conservation and divergence in brain patterning and morphogenesis throughout ontogenesis and evolution.

\section{MATERIALS AND METHODS}

\section{Animals}

Feline (Felis catus) embryos or fetuses were obtained from domestic cats referred to the Unit of Obstetrics and Surgery of the Companion Animal Clinic of the School of Veterinary Medicine, Faculty of Health Sciences, Aristotle University of Thessaloniki. More specifically, clinically healthy female cats admitted to the Unit for preventive ovariohysterectomy were screened; in case of pregnancy, the excised gravid uterus was not processed for incineration, but immediately incised. The anesthetic protocol included: premedication with combined dexmedetomidine $\left(25 \mu \mathrm{g} \mathrm{kg}^{-1} \mathrm{im}\right)$ and butorphanol $(0.1 \mathrm{mg}$ $\left.\mathrm{kg}^{-1} \mathrm{im}\right)$, analgesia with meloxicam $\left(0.1 \mathrm{mg} \mathrm{kg}^{-1} \mathrm{sc}\right)$, anesthesia induction with ketamine (10 $\left.\mathrm{mg} \mathrm{kg}^{-1} \mathrm{im}\right)$, and maintenance with ketamine (4-6 $\mathrm{mg} \mathrm{kg}^{-1} \mathrm{iv}$ ). Standard operational procedures were followed for midline approach ovariohysterectomy.

Embryos (or fetuses) were collected, rinsed in cold Phosphate Buffer Saline (PBS), and were either immediately frozen $\left(-80^{\circ} \mathrm{C}\right.$; tissues intended for RNA/DNA extraction), or fixed with $4 \%$ w/v paraformaldehyde (PFA) in PBS for (at least) $24 \mathrm{~h}$ at $4^{\circ} \mathrm{C}$. Following fixation, tissues were washed with PBS, cryoprotected in $30 \% \mathrm{w} / \mathrm{v}$ sucrose in PBS, embedded in the appropriate sectioning plane (coronal, horizontal, or sagittal) using Tissue Freezing Medium (Leica, Germany), and stored at $-80^{\circ} \mathrm{C}$ until sectioning. Sections $(12 \mu \mathrm{M})$ were generated using a Leica CM1900UV cryostat, collected on Superfrost plus (Fisher Scientific, United States) slides, air-dried for at least $30 \mathrm{~min}$, and stored at $-80^{\circ} \mathrm{C}$ until later use.
Gestational age was initially assessed during the pre-surgical physical examination, as well as by examining macroscopically the gravid uterus. Given this first approximate estimation, embryos and fetuses were staged after fixation, according to their morphology and crown-rump length (Evans and Sack, 1973; Knospe, 2002). As embryos and fetuses were not collected from timed pregnancies, crown-rump length measurements and morphology do not allow for the accurate evaluation of the gestational age. Thus, we grouped embryos and fetuses in classes spanning two consecutive days, as follows: E22/23 ( $n=7), \mathrm{E} 24 / 25$ $(n=12), \mathrm{E} 26 / 27(n=21), \mathrm{E} 28 / 29(n=3)$ and $\mathrm{E} 34 / 35(n=5)$.

\section{Riboprobes}

The DNA fragments used for the generation of riboprobes were amplified either from genomic DNA (gDNA) or from E26/27 feline brain complementary DNA (cDNA). Primers (Supplementary Table 1) were designed using the National Center for Biotechnology Information (NCBI) Primer Blast tool ${ }^{1}$, based on the feline genomic/cDNA sequences published in GenBank. Amplicons were cloned (following suitable digest to create "sticky" or "blunt" ends, or further minor modifications; for details, please refer to the Supplementary Table 2) in pBluescript II KS(+) (Agilent/Stratagene, United States). All constructs were verified by sequencing (Starseq, Germany). Linearized plasmids were used for in vitro transcription of antisense RNA probes with T3 or T7 RNA polymerase (Takara, Japan), according to manufacturer's instructions, using Digoxigenin-11-UTP (Roche, Switzerland). For Nkx2-1, we used the murine probe (Shimamura et al., 1995), given that the identity between the murine and feline homologs is high (96\%).

\section{In situ Hybridization}

In situ hybridization was performed as previously described (Stylianopoulou et al., 2016). Slides were mounted in Glycergel (DAKO) and photographed with a Leica DM5500 B (Leica Microsystems) microscope equipped with a DFC7000T or a DFC310FX digital camera (Leica Microsystems). Images were captured using the camera software (LAS v4.13, Leica Microsystems); image panels and schemata were created with the GIMP (gimp.org).

\section{RESULTS}

\section{Timing of Patterning and Cell Specification in the Feline Telencephalon}

In murine embryos, patterning and cell specification events mainly occur between E11 and E14.5 (for review please refer to Guillemot, 2005; Martynoga et al., 2012), while most major genoarchitectonic studies (e.g., Puelles et al., 2000, 2016a; Medina et al., 2004; Flames et al., 2007; García-López et al., 2008; Abellán et al., 2014) have been performed in E12.5-13.5 embryos. As already mentioned in the materials and methods, the feline embryos and fetuses used in this work were obtained from domestic cats and the gestational age was assessed according to

\footnotetext{
${ }^{1}$ https://www.ncbi.nlm.nih.gov/tools/primer-blast
} 
their morphology and crown-rump length (Evans and Sack, 1973; Knospe, 2002). To define the developmental stages that would correspond to the murine E12.5-13.5; we used the prediction model of neural development developed by Workman et al. (2013). Key events (Supplementary Table 3) of the development of the cortex, the limbic system, and the striatum were used to translate developmental time between mouse and cat embryos. The model predictions indicated a rough equivalence of the murine stages E11, E12, and E13/14 to feline E22/23, E24/25, and E26/27, respectively, these results were further corroborated by studying the general morphology of the feline brain on sections. Given that two earlier works (Luskin and Shatz, 1985a,b) on feline embryos and fetuses had demonstrated that the first neurons within the pallium are generated between E24 and E30, we chose to focus primarily on the E26/27 feline embryonic telencephalon. Embryos of earlier (E24/25 and E22/23) or fetuses of more advanced (E28/29, E34/35) developmental stages were occasionally used for corroboration purposes and to temporally map developmental events.

We then utilized in situ hybridization with markers for various telencephalic domains that have been established in other species (mainly in the mouse) namely: Lhx2, Lef1 (Abellán et al., 2014), Emx1, Tbr1, Pax6 (Puelles et al., 2000), Nr4a2 (Nurr1; Puelles et al., 2016a) Tbr2 (Eomes; Englund et al., 2005), Dlx2, Nkx2-1, Lhx6, Lhx7, Er81 (Sussel et al., 1999; Flames et al., 2007; García-López et al., 2008), Gad2 (Katarova et al., 2000), and Mash1 (Casarosa et al., 1999). To this end appropriate gene segments (Supplementary Table 2), were amplified by PCR (Supplementary Table 1) from feline gDNA or cDNA (transcribed out of E26/27 head total RNA) and cloned into suitably prepared vectors. Recombinant plasmids were used to generate DIG-labeled riboprobes through in vitro transcription. The analysis of the results was performed using published atlases (Jacobowitz and Abbott, 1997; Schambra, 2008; Paxinos and Ashwell, 2018) and articles (Medina et al., 2004; Flames et al., 2007; García-López et al., 2008; Abellán et al., 2014; Puelles et al., 2016a), as well as online resources (Allen Brain Atlas²; GenePaint $)^{3}$ on the developing mouse or rat brain.

\section{The Pallium and the Subpallium}

To delineate the proliferative zones of the pallium and the subpallium, along the rostrocaudal axis, we compared the expression patterns of Tbr2 and Dlx2 in E26/27 embryos. In the mouse, the pallial proliferative zones strongly express $T b r 2$ (Bulfone et al., 1999), a characteristic attributed to the population of basal intermediate progenitors of glutamatergic neurons that constitute the pallial svz (Englund et al., 2005); Dlx2 is strongly expressed by the populations of the (non-glutamatergic) progenitors of the subpallium, demarcating, thus, the extent of the subpallial svz/vz (Eisenstat et al., 1999). In several studies Pax6 or Tbr1 have been used to demarcate the pallium and Gs 22 or Dlx2 the subpallium (Puelles et al., 2000; Yun et al., 2001; Stenman et al., 2003b; Carney et al., 2009; Desfilis et al., 2018); we chose, however, Tbr2 over Pax6 as the latter expands into the subpallial striatal division (Yun et al., 2001; Flames et al., 2007),

${ }^{2}$ https://developingmouse.brain-map.org/

${ }^{3}$ https://gp3.mpg.de/ hampering the delineation of the pallial domain. Moreover, we chose Dlx2 over Gsx2, as the former is expressed by cells residing in both proliferative zones (Eisenstat et al., 1999). Tbr1, on the other hand, is a well-known marker of pallial post-mitotic neurons (Bulfone et al., 1995; Englund et al., 2005), we, therefore, considered that we cannot use it as we could not perform a direct comparison of its expression with the $D l x 2$ expression pattern.

Rostrally, around the coronal level where the fila olfactoria are visible, almost the whole telencephalon expressed Tbr2, apart from a lateroventral, spot-like, Tbr2-negative, region that showed high Dlx2-expression (Figures 1A,B); this suggested that rostrally, the telencephalon was genoarchitectonically pallial, except for a well-demarcated focus in the lateral-ventral wall corresponding to the rostral-most end of the striatal partition. More posterior planes revealed that the Dlx2-expressing territory expanded ventrally toward the medial telencephalic wall (the septal division of the striatal partition; compare Figures 1A,B, 2B,C and Supplementary Figures 1A, B) at the expense of the Tbr2 expression domain. Furthermore, Dlx2 was highly expressed in the proliferative zones of the central and amygdaloid divisions of the subpallial partitions (ganglionic eminences) while Tbr2 was confined dorsally, demarcating the pallial anlagen (Figures 1C-H). Noticeably, the caudalmost end of the ventral telencephalon expressed high levels of Tbr2 and presented Dlx2-expression only in its dorsal-most tip (Figures 1I,J, for close-ups, see Figures 3E,F,K,L). This domain corresponded to the ventropallial amygdalopiriform area (VAP), while the Dlx2 (Figures 1I, 3K) expressing area was considered to correspond to the vz of the striatal amygdala (AStr).

The mutually exclusive expression of Tbr2 and Dlx2 along with Tbr1 and Gad2, assisted us in locating the pallialsubpallial boundary (PSB), in the following locations: (1) In the lateral telencephalic wall, all along the rostrocaudal axis (Schemata in Figures 2-4); (2) In the medial telencephalic wall (septal PSB), caudal to the retrobulbar area, but rostral to the plane of the interventricular foramina (ivf) (Figures 2B,C and Supplementary Figures 1A,B); (3) In the medial aspect of the basal telencephalon, caudal to the positive prethalamic eminence (PThE), between the amygdaloid subpallial division and the VP (or CVP according to Ruiz-Reig et al., 2018; Figures 3D,J,M,E,K,N); (4) In the caudal-most aspect, between the VAP and the AStr (Figures 3F,L,O). As in mice and chicken (Puelles et al., 2000), the PSB could be imagined as a plane connecting the ventricular and pial surfaces, spanning from the Tbr2/Dlx2 abutting vz/svz region to the Tbr1/Gad2 neighboring territory of the lot.

\section{The Tetrapartite Nature of the Feline Pallium}

To delineate molecularly distinct partitions within the feline pallium we studied the combinatorial expression of Tbr2, Emx1, Pax6, Lhx2, Lef1, Tbr1, and Nr4a2. Our analysis was based on the tetrapartite model described by Puelles et al. (2000, 2016a).

\section{Ventral Pallium}

The feline VP was molecularly characterized by the presence of Tbr2 (with a salt-and-pepper pattern), Pax6 (strong expression), and $L h \times 2$ (weak expression) in the vz, which lacked Emx1, 


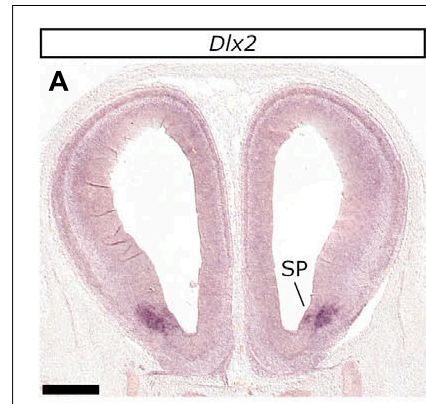

c

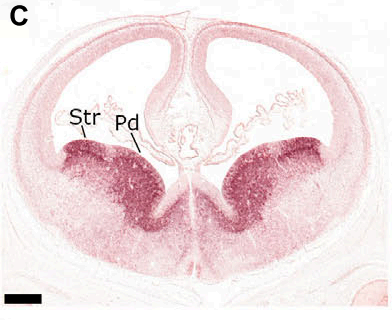

E
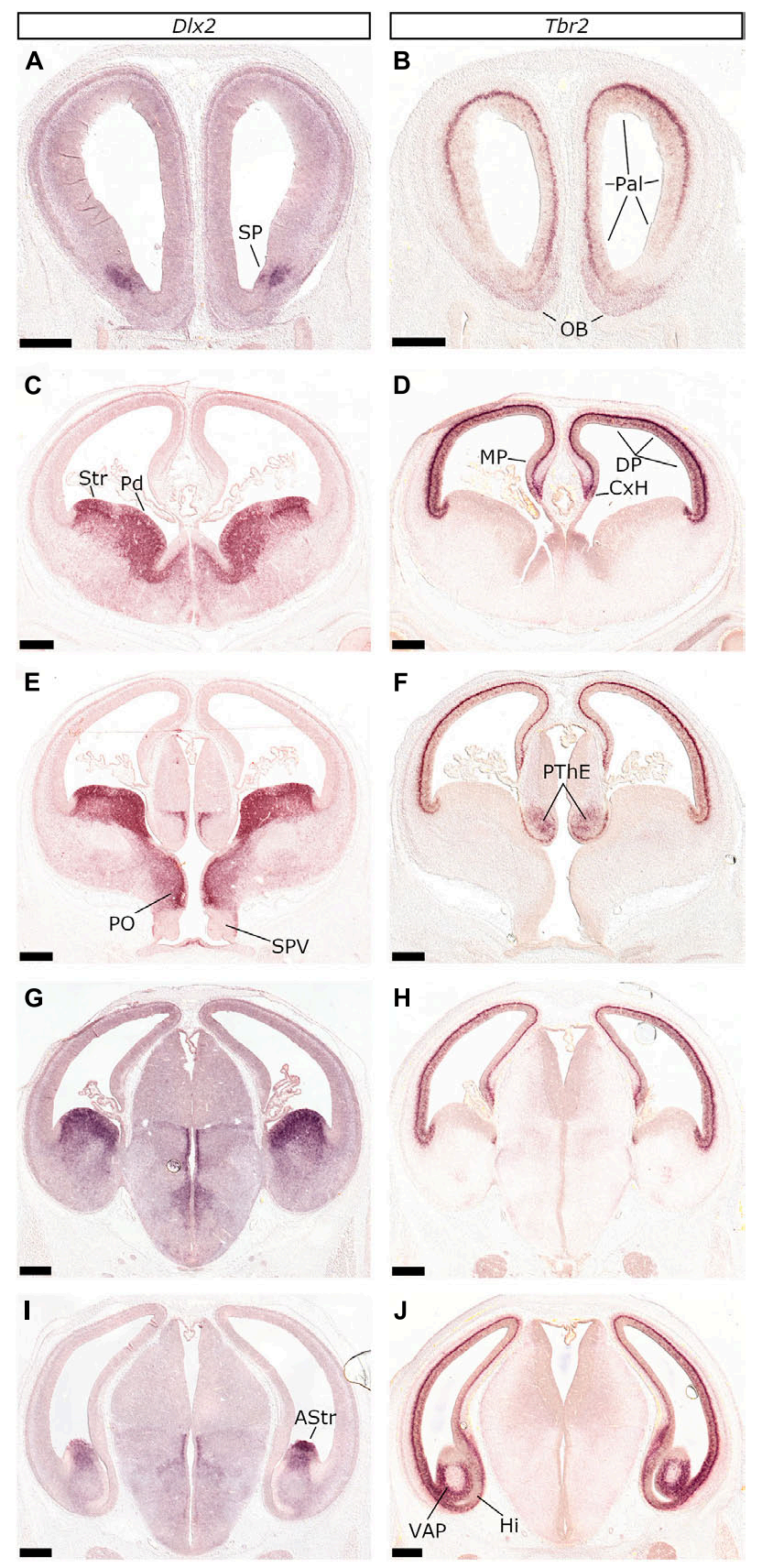

D
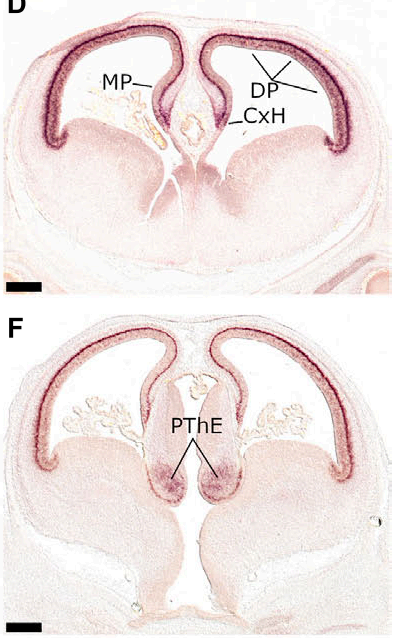

H

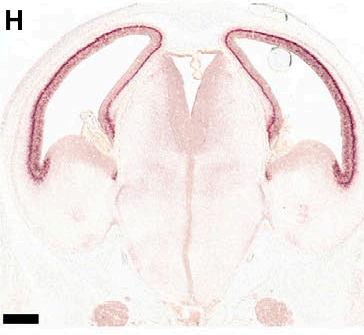

$\mathbf{J}$

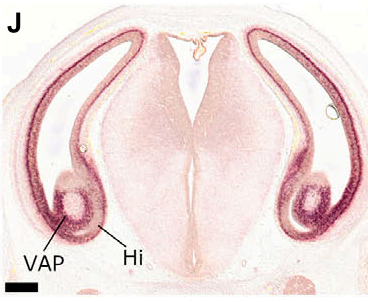

FIGURE 1 | Tbr2 and D/x2 expression demarcate the proliferative zones of the feline pallium and subpallium along the rostrocaudal axis. In situ hybridization on coronal sections of E26/27 embryos with Tbr2 (A,C,E,G,I) or Dlx2 (B,D,F,H,J) probes. Scale bars $500 \mu \mathrm{m}$.

Tbr2 (strong expression) and Emx1 (weak expression, barely visible in Figure 2A) in the svz, and Tbrl and Emx1 in the cortical plate (cp) (Figures 2, 3A-F, 4A-D,I). The analysis of rostral sections revealed that apart from the lateral telencephalic wall (Figures 2A, 3A, 4B and respective schemata) a small region within the medial-ventral (septal) proliferative zones also displayed the VP molecular profile (strong Pax6 labeling of

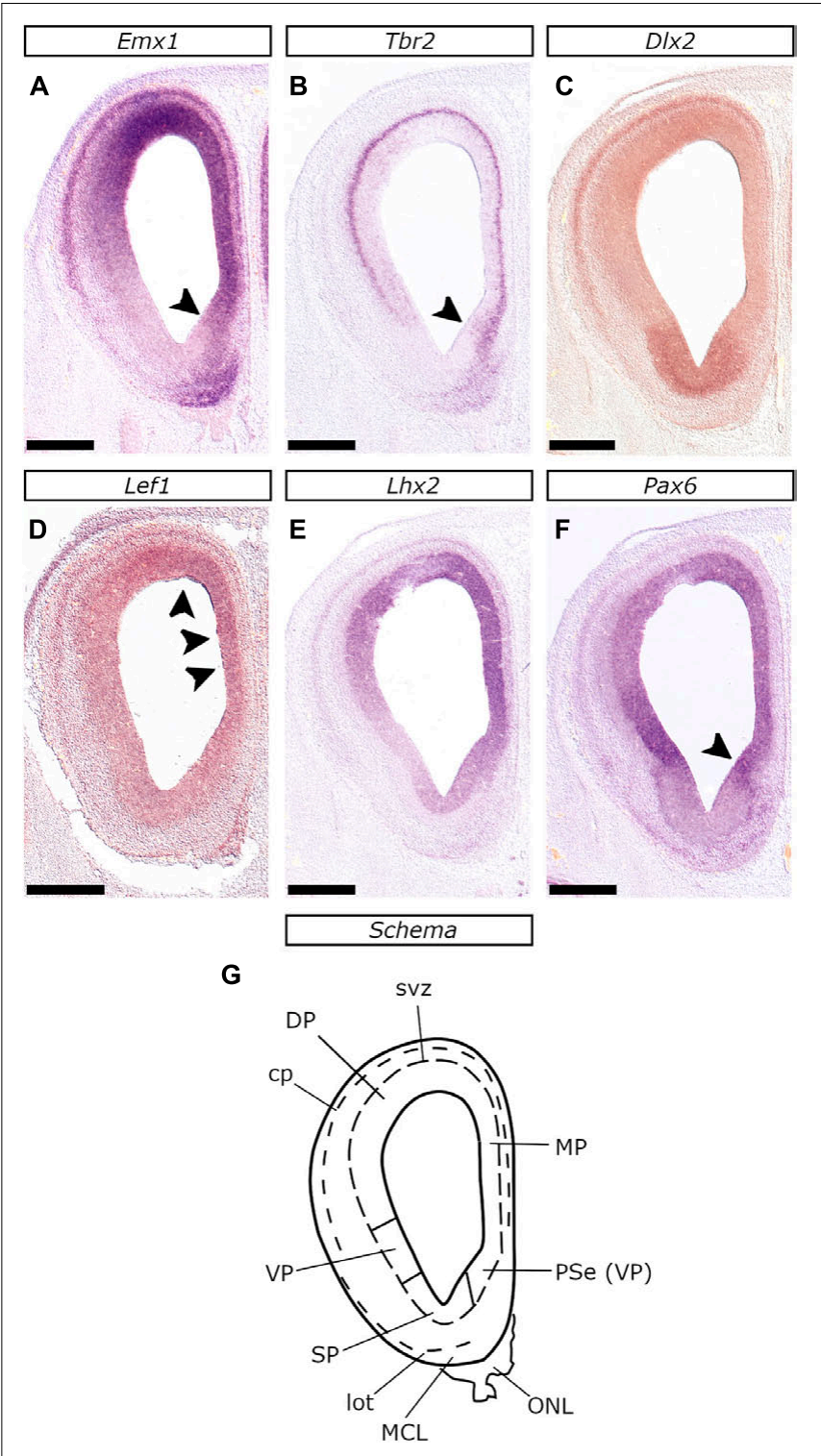

FIGURE 2 | Organization of the E26/27 telencephalon at the retrobulbar level. In situ hybridization on coronal sections with $E m \times 1$ (A), Tbr2 (B), D/x2 (C), Lef1 (D), Lhx2 (E), and Pax6 (F) probes. (G) Schematic representation of the plane of section corresponding to the coronal sections shown in (A-F). The ventricular zone (Vz) of the ventral pallium (VP) expressed high levels of Pax6, low levels of $L h x 2$, but lacked $E m \times 1$ expression (compare $\mathbf{F}, \mathbf{E}$, and $\mathbf{A}$ ). Arrowheads in $\mathbf{( A , B , F )}$ indicate the ventral region of the pallial septum (PSe) that expressed high levels of Pax6 (F) and Tbr2 (B), along with low levels of Emx1; this region was considered to be part of the VP. Arrowheads in (D) indicate Lef1 expression in the vz of the dorsal and the medial (dorsal-most region) telencephalic wall. Note the expression of Emx1 and Tbr2 within the mitral cell layer (MCL). Scale bars $500 \mu \mathrm{m}$.

the weakly Emx1-expressing vz; arrowheads in Figures 2A,B,F, Supplementary Figure $\mathbf{5}$ and respective schemata); this implied that the VP extends across the rostral ventral telencephalon, including the olfactory bulb, from the lateral to the medial wall, as anticipated by the concentric ring topology (Puelles et al., 2019). In caudal planes, posterior to the internal capsule, 


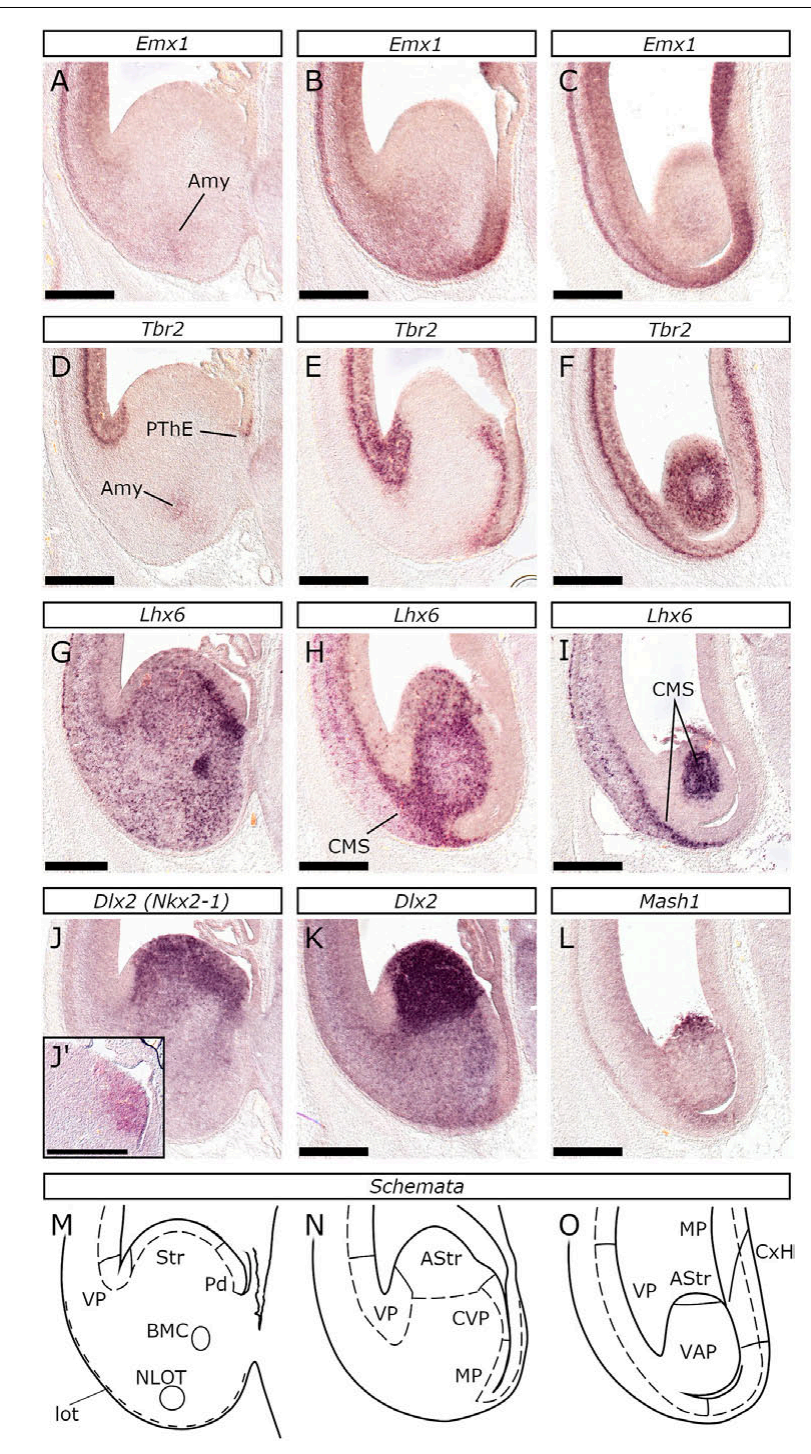

FIGURE 3 | At posterior levels, VP expanded at the expense of subpallium which became gradually restricted. In situ hybridization at three successive coronal levels of E26/27 embryonic brain posterior to the internal capsule. At

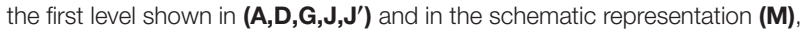
the VP could be identified in the lateral wall by Tbr2 expression in the sVZ and the $\mathrm{vz}$ (D) and the absence of Emx1 in the $\mathrm{vz}(\mathbf{A})$. The subpallium consisted at this level of both Str and Pd domains, as indicated by Nkx2-1 expression in the vz/svz $\left(\mathbf{J}^{\prime}\right)$ and $L h x 6$ in the svz (G) within the medial aspect of the D/x2 labeled $(\mathbf{J})$ domain. Tbr2 expression was also observed in the prethalamic eminence (PThE), while both Tbr2 and Emx1 expression marked the amygdala primordium (Amy in A and D). Lhx6 expression further demarcated the basal magnocellular complex (BMC) while the nucleus of the lateral olfactory tract (NLOT) primordium appeared as a superficial Lhx6-negative

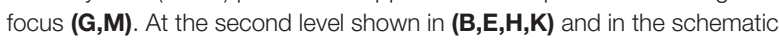
representation $\mathbf{( N )}$ the pallium extended in the medial aspect of the ventral telencephalon and the subpallium restricted. Tbr2 expression was observed in both proliferative zones of this pallial domain (E); Emx1 expression appeared to divide it into a dorsal Emx1-negative and a ventral Emx1-expressing subdomain (B). The dorsal area corresponds to the central VP (CVP), while the ventral represents the MP (N). At this level, Nkx2-1 was not expressed (data not shown), thus the caudal subpallium is striatal in nature corresponding to the AStr (N). Lhx6-expressing neurons $\mathbf{( H )}$ exit the ventral

(Continued)
FIGURE 3 | telencephalon and migrate through the pallial svz/iz; this represents the caudal migratory stream (CMS). At the third level shown in $\mathbf{( C , F , I , L )}$ and in the schematic representation $\mathbf{( O )}$ the entire ventral telencephalon expressed Tbr2 (F) but not $E m \times 1$ (C) and it corresponded to the vz of the VAP. The subpallium (AStr) was restricted to the dorsal-most tip, as revealed by Mash1 expression (L). The core of the VAP showed high Lhx6 expression (I), which is attributed to the migrating interneurons of the CMS. Scale bars $500 \mu \mathrm{m}$.

the Tbr2- PThE was continuous with a small Tbr2 expressing pallial territory, which gradually grew, ultimately extending into the CGE (Figures 3D-F, see also Figures 5B,F,J). The careful examination of adjacent sections revealed that the $\mathrm{vZ}$ of this compartment apart from Tbr2, presented strong Pax6labeling (Figures 5A,E,I), weak $L h x 2$ expression (Figure 5K), being otherwise, devoid of Emx1 signal (Figures 3B,C,5G). We considered this compartment to correspond to the CVP (RuizReig et al., 2018). The VP expression profile described above at E26/27 was evident from the earliest stage examined, E22/23 (Figure 6). Interestingly, the VP was the only pallial sector that expressed Tbr2 (with a "salt-and-pepper" pattern; Figures 7A-D and close-up in Figure 6C) at E22/23 (although Tbr2 labeling of the LP vz could not be ruled out).

The VP mantle exhibited (strong) Tbr1, (moderate) Emx1, and (weak) $L h x 2$ expression, associated mainly with the $\mathrm{cp}$. The latter was obvious as a Tbr1 positive stratum at E22/23 (Figure 6A), E24/25 (Figure 6G), E26/27 (Figure 4I), E28/29 (Figure 8A), and E34/35 (Figure 8C). Furthermore, Tbr1 expression at E22/23, followed the characteristic pattern described in the mouse (Medina et al., 2004), gradually diminished in the DP (it was unclear however if the medial pallial mantle expressed Tbr1 at this stage, or if its levels were below the detection limit of our technique; compare the expression of Tbr1, Tbr2, and Emx1 in Figures 7A-D). Given the role of Tbr1 in the neurogenesis of early-born glutamatergic neurons (Hevner et al., 2001), we assumed that in the cat, the first pallial post-mitotic neurons arise around E22/23 in the VP mantle.

\section{The Medial Pallium}

At E26/27, Lef1, Lhx2, Emx1, Tbr2, and Pax6 expression characterized the MP proliferative zones, while Tbr1, Lhx2, Emx1, and Lef1 transcripts appeared in the MP mantle. Our analysis was particularly based on the pattern of Lef1, given its selective expression within the MP (Abellán et al., 2014) and its role in hippocampal development (Galceran et al., 2000). At rostral levels of the E26/27 embryonic forebrain, weak Lef1 expression was observed in the vz of the dorsal-most telencephalic wall and the dorsal aspect of the medial telencephalic wall (arrowheads in Figure 2D). In more caudal planes, the Lef1-positive territory covered the entire medial telencephalic wall, expanding to the pallial septum and slightly into the DP (Figures 4C, 9I,O). Lef1expression in the vz of E22/23 embryos expanded far beyond the MP, into the rest of the pallial sectors, almost reaching the VP (with weaker labeling than in the MP though; Figure 9C). At E24/25 however, the Lef1 expression domain in the DP appeared smaller (Figures 9F,L) in comparison to that of E22/23 (Figure 9C), while in E26/27, Lef1 covered, apart from the 


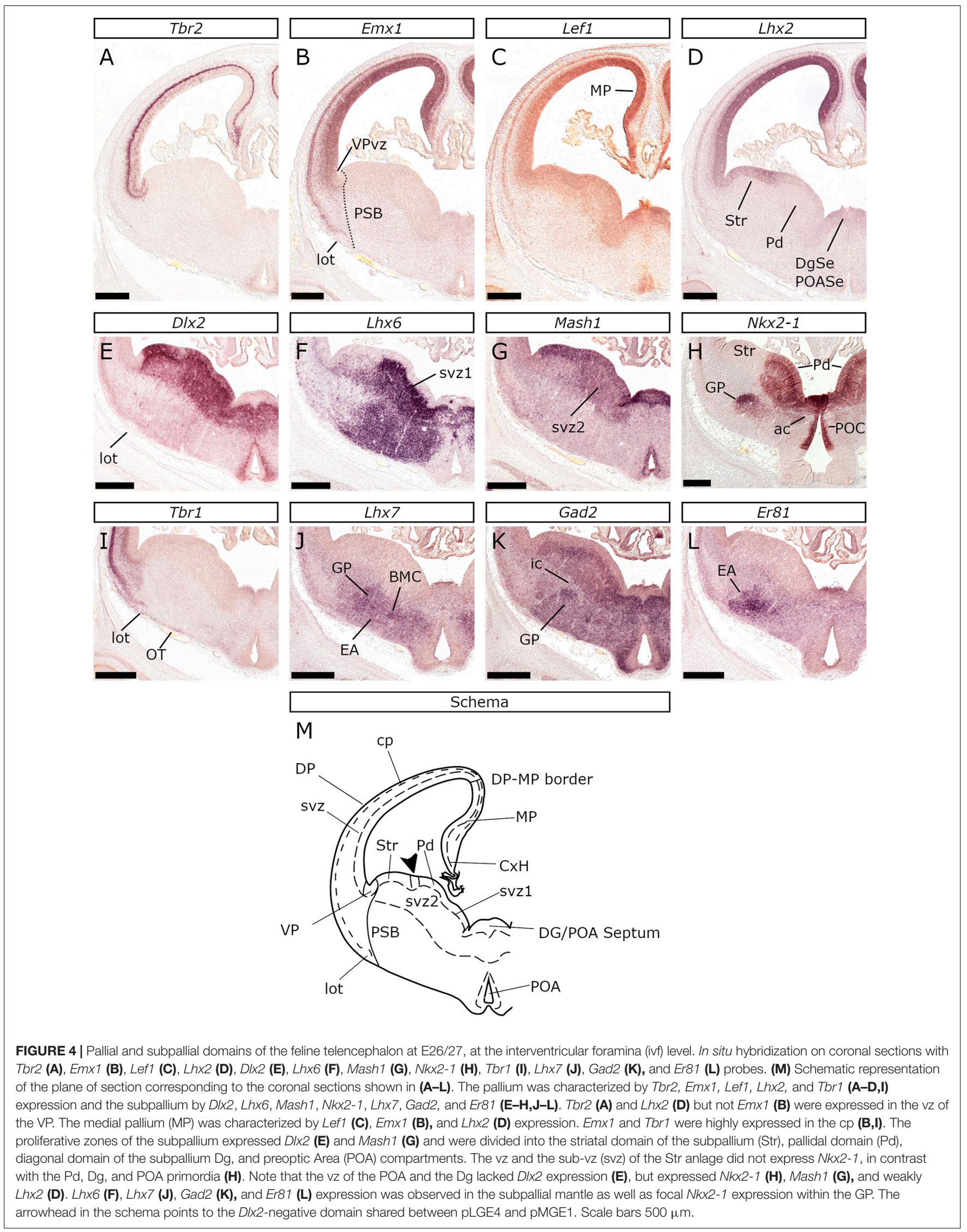



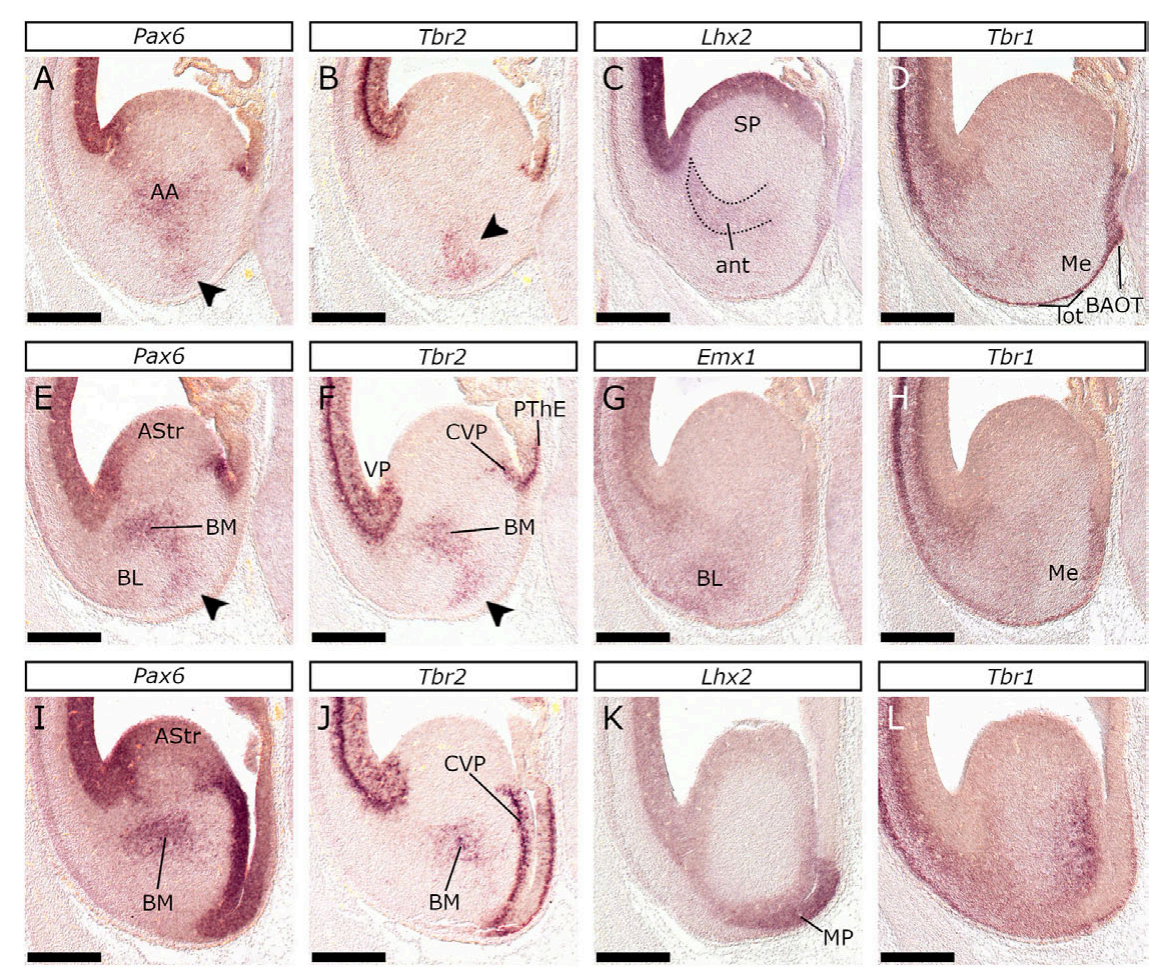

FIGURE 5 | Nuclear organization of the amygdala at E26/27. In situ hybridization in three successive coronal planes of the E26/27 embryonic brain with Pax6

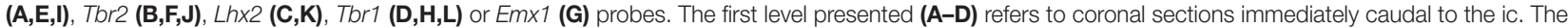
presumed anterior amygdalar radial unit (dotted area in $\mathrm{C}$ ) expressed $L h \times 2$. Pax6 was expressed in the AA primordium (A), while more diffuse Pax6 labeling was also observed superficially (arrowhead in A), in an area that was further characterized by Tbr2 expression (arrowhead in B). This area is in close association with the lot, that expressed Tbr1 along with the BAOT primordium (D). Notably, the Me anlage lacked Tbr1 expression (D). Caudal to this level, Pax6 (E) and Tbr2 (F) were expressed in the BM primordium and the superficial domain (arrowheads) as noted in (A,B). Notably, the Pax6/Tbr2 expressing area embraced the Emx1-expressing BL primordium (G). The superficial domain is thought to represent the ACo primordium, or subpopulations of the Tbr1-negative (H) Me primordium. At more caudal planes the Pax6/Tbr2-expressing focus represents the BM anlage (I,J). Note that the pallial vz ventral to the CVP expressed Lhx2 (K) corresponding to the MP. Scale bars $500 \mu \mathrm{m}$.

pallial septum, only the MP-abutting DP vz (Figures 9I,O). An interesting feature of the MP at E26/27 was that, in contrast with the other pallial divisions, it was mostly devoid of vz-associated Tbr2 expression (Figures 10E,F,H; see also Figure 4A). However, the medial most region of the telencephalic wall, just adjacent to the choroid tela, displayed a patchy expression pattern of Tbr2 (Figures 10F,H) in the vz, which was devoid of Emx1 (Figure 9M), Lhx2 (Figure 10G) and Pax6 (Figure 10I). Apart from E26/27, this was also observed at E22/23 (Figures 9A, $\mathbf{A}^{\prime}, \mathbf{B}$ ) and E24/25 (Figures 10A-C); based on the genoarchitectonic profile described and its proximity to the choroid plexus, this part was considered to represent the cortical hem $(\mathrm{CxH})$.

\section{The Dorsal and Lateral Pallia}

Regarding the DP and the LP, there is, to our knowledge, no single marker that exclusively labels their proliferative zones. Differential analysis of several gene expression patterns in the feline pallium revealed, however, that at E26/27 the DP expressed Emx1, Lhx2, Pax6, and Tbr2 throughout its proliferative zones (Figures 2, 4), and Tbr1 (data not shown), Emx1 (Figure 4B), and (weak) Lhx2 (Figure 4D) in the cp. As already mentioned, the vz of the DP domain that neighbored the MP, showed weak Lef1 expression. This molecular profile was further used to demarcate the DP in E22/23 and E24/25 embryos (Figures 6, 7, 9). In mice, the LP vz is interposed between the Emx1/Lhx2-enriched DP and the Emx1-negative VP, and it is characterized by weak Emx1 and Lhx2 expression (Puelles et al., 2000; Abellán et al., 2014; Desfilis et al., 2018). In our experiments, however, the LP vz was not clearly demarcated in any of the stages studied as we did not detect sharp boundaries between the expression domains of the aforementioned genes. Based on the Puelles et al. (2016a) approach, however, we identified the LP mantle in E28/29 embryos, as an Nr4a2-labeled domain (Figures 8B,F) within the Tbr1-expressing region (Figures 8A,E). At E34/35, Nr4a2-labeling demarcated the claustral domain (Figures $\mathbf{8 D}, \mathbf{H}$ ), the dorsal endopiriform nucleus (ventralward migrating populations, Dorsal Endopiriform Nucleus (EPd) in Figure 8D and arrowhead in Figure $\mathbf{8 H}$ ) as well as dorsalward migrating cells entering the isocortex (A in Figure 8H). Notably, Nr4a2 expression was not detected in the claustral primordium (and in general in the pallium) of E26/27 and earlier embryos. The subpallial mantle however showed weak Nr4a2 labeling in an area coexpressing Er81 (Supplementary Figure 2) and other subpallial genes (data not shown). 


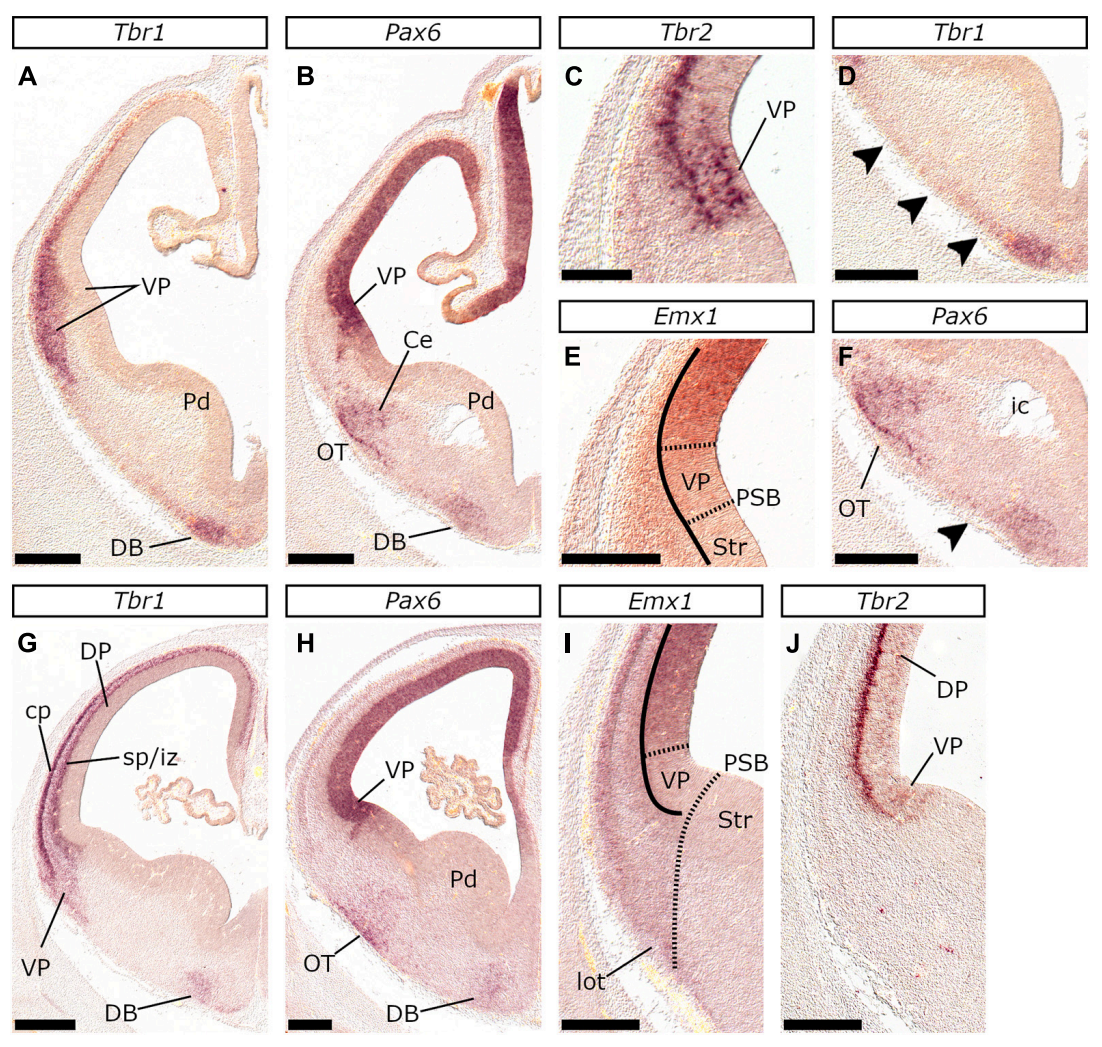

FIGURE 6 | Tbr1 in the VP mantle was expressed at E22/23 but the lamination of the $\mathrm{cp}$ and the sp/iz appeared at E24/25. In situ hybridization on coronal sections at E22/23 (A-F) and E24/25 (G-J) with Tbr1 (A,D,G), Pax6 (B,H), Tbr2 (C,J) and Emx1 (E,I) probes. At E22/23 Tbr1 appeared first in the VP mantle with a VP-high to DP-low gradient (A). Pax6 (B) and Tbr2 (C) were expressed in the vz of the VP, in contrast with Emx1 (E). Tbr1 was also present in the diagonal band (DB) anlage in the subpallium (A,D), along with Pax6 (B,F). Arrowheads in (D,F) indicate a migrating, septal-derived, cell population, that expressed low levels of Tbr1 and Pax6 and extended subpially between the DB and the VP. Pax6 expression was detected further in OT (B,F). At E24/25, Tbr1 (G) expression marked the cp and the sp/iz, revealing the lamination of the pallial mantle in contrast with E22/23 (compare $\mathbf{A}$ with $\mathbf{G}$ ). Lamination was more obvious at the VP-LP mantle; cp and sp/iz converge at the MP and the dorsal-most regions of the DP to a single Tbr1-expressing band. At E24/25 the vz of the VP was still Tbr2 positive (J) but lacked Emx1 expression (I). Notably, Tbr2 expression was extended beyond the VP, into the vz of the DP (compare C and J). Scale bars $500 \mu \mathrm{m}$, except for (C) and (E): $250 \mu \mathrm{m}$.

\section{Major Compartments of the Subpallium}

To delineate molecularly distinct territories within the feline subpallium we studied the expression of Dlx2, Mash1, Nkx2-1, Er81, Pax6, Lhx2, Lhx6, Lhx7, and Gad2 that have been used in similar studies in other vertebrates. We analyzed our results according to the developmental ontology (Puelles et al., 2013, 2016b; Watson et al., 2017).

\section{The Subpallial Proliferative Zones}

\section{The Striatum}

The vz of the feline striatal anlage was molecularly characterized by Dlx2 (Figures 4E, 11A,B,I,M), Mash1 (Figures 4G, 11E,K,O) and $\operatorname{Lh} \times 2$ (Figure 4D) expression but not by $N k \times 2-1$ (Figures 4H, 11H,L,S). Pax6 was also detected in the striatal vz, but with a lateral-high to a medial-low gradient of expression (Figures 11F,R; present also on the septal striatum, arrowhead in Figure 7F). In the striatal svz, Dlx2 (Figures $4 \mathrm{E}, 11 \mathrm{~A}, \mathrm{~B}, \mathrm{I}, \mathrm{M}$ ) and Gad2 (Figures 4K, 11G,P) were detected. Lhx6 expression was also observed in a "salt and pepper" pattern (Figures 4F, 11J,N) in the tangentially migrating, pallidal-derived, interneurons, en route to the pallium (Liodis et al., 2007). The dorsal-most aspect of the feline striatal svz was characterized by a strong focal expression of Pax6 (Figure 11F) and Er81 (Figure 11D). Interestingly, Er81 was also weakly expressed in a small part of the dorsal-most striatal vz (Figure 11D) overlying the strongly Er81/Pax6-positive svz area; thus, the feline striatal anlage proliferative zones were divided into two subdivisions, a dorsal and a ventral. Ventrally, the striatal vz showed a spot devoid of Dlx2, but with high Mash1 and Lhx2 expression (compare Figures $4 \mathrm{D}, \mathrm{E}, \mathrm{G}, \mathbf{1 1} \mathrm{I}, \mathrm{K}, \mathbf{M}, \mathbf{O})$. In mice this area is associated with the Nkx6-2-expressing, intereminential sulcus region shared between the progenitor domains pLGE4 and pMGE1 (Flames et al., 2007); accordingly, the pLGE4 in the cat is an Lhx2-positive (Figure 4D), Mash1-positive, but Dlx2-negative domain within the ventral striatal anlage. The striatal subdivision extended throughout the rostrocaudal axis, from the lateralventral telencephalic wall rostrally (Figure 1A), to the dorsal tip of the caudal-most end of the amygdaloid division caudally (Figures 1I, 3J-L). However, as in mice (Flames et al., 2007), the Dlx2-negative pLGE4 domain did not extend caudally to the level of the internal capsule (compare Figures 3J,K, 11M). Notably, in caudal levels (Figure 5I), the subpallial svz showed 

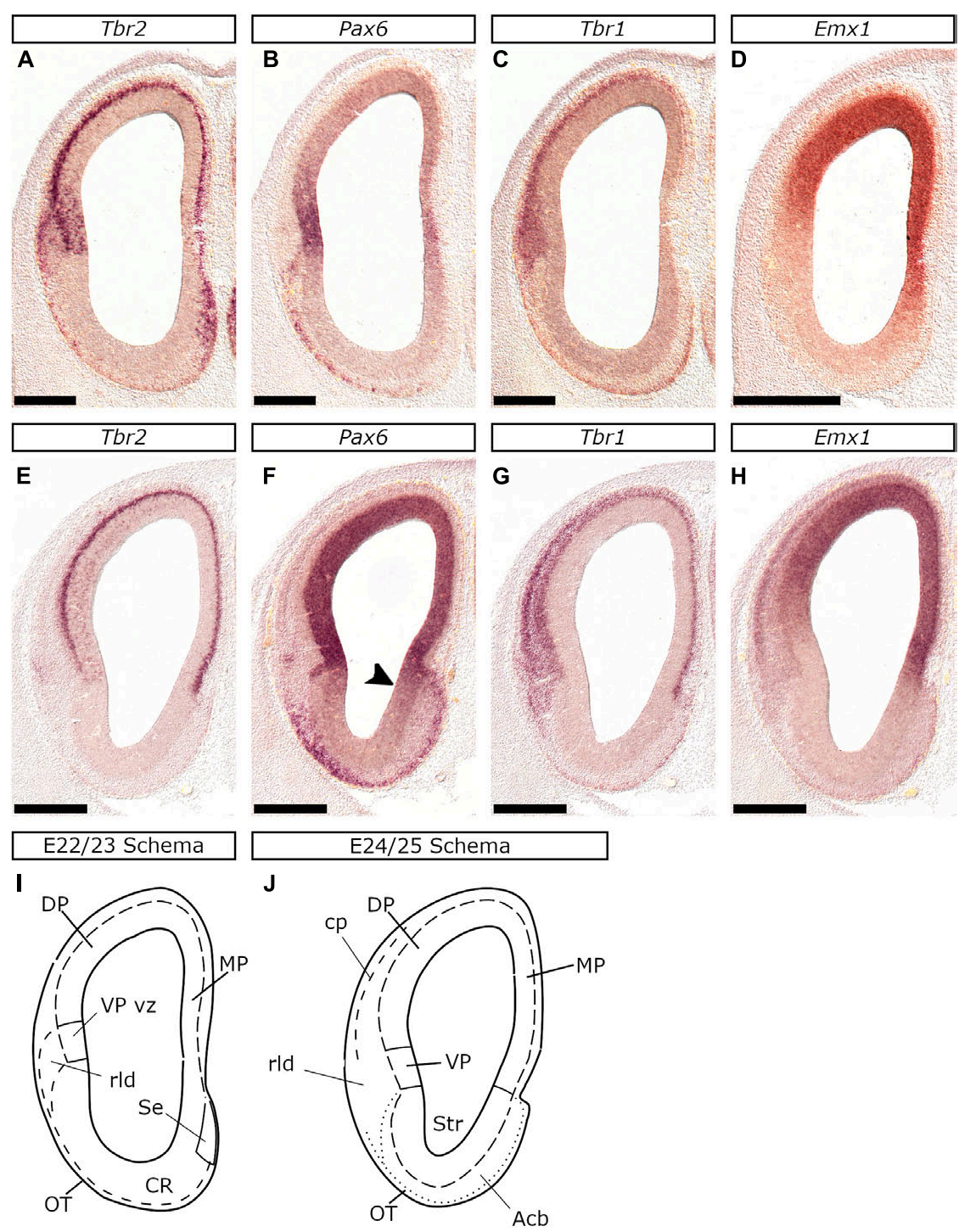

FIGURE 7 | Cajal-Retzius neurons in the retrobulbar telencephalic fields at E22/23 and E24/25. In situ hybridization on coronal adjacent sections of E22/23 (A-D) and E24/25 (E-H) with Tbr2 (A,E), Pax6 (B,F), Tbr1 (C, G) and Emx1 (D,H) probes. (I,J) Schematic representations of the coronal levels corresponding to (A-D) or (E-H) respectively. Tbr2 (A) apart from the pallial svz and the vz of the VP, was further expressed by postmitotic neurons in the Septum (Se) and the VP mantle (rld) which are both known sources of CR neurons. Note that, the Tbr1-expressing subpial stream of CR neurons in (C), expressed also Tbr2 (A,I). At E24/25, Tbr1 was still detected subpially in the basal telencephalon (G), though at lower levels than in E22/23. Note the difference in the Tbr1 expression pattern: at E22/23 Tbr1 transcripts labeled diffusely the pallial mantle (C) however at E24/25 the cp could be clearly detected as a sharply Tbr1-expressing layer (G). Pax6 was expressed within the mantle of the VP-LP (rld) both at E22/23 (B) and E24/25 (F), in neurons migrating toward the OB, as well as in a stream emanating from the dorsal striatal vz toward the olfactory tubule (OT). Arrowhead in (F) indicates the dorsal part of the (subpallial) septal striatal vz that was characterized by a prominent Pax6 gradient indicating that the dorsal (subpallial) septum shares the same molecular identity with the dorsal central striatal subdivision. Dotted line in ( $\mathbf{J}$ ) marks the subpial migration of Pax6-expressing cells from the dorsal striatal subpallium (central or septal), toward the nucleus accumbens (Acb) and the OT. Scale bars $500 \mu \mathrm{m}$.

Pax6 expression both in its lateral (VP-abutting) and medial aspect (CVP-abutting), suggesting that the AStr bears the dorsal striatal molecular profile.

\section{The Pallidum}

The vz of the pallidal anlage featured strong Mash1 (Figures 4G, 11K), Dlx2 (Figures 4E, 11I), and $N k x 2-1$ (Figures 4H, 11L) labeling, being however is devoid of Lhx2 (Figure 4D) and Pax6 (Figures 11F,R) expression; the svz expressed Lhx6 (Figures 11J,N), Dlx2 (Figures 4E, 11I), Nkx2-1 (Figures 11L,S), Gad2 (Figures 4K, 11P), and Mash1 (Figures $11 \mathrm{~K}, \mathbf{O}$, particularly the svz2, in contrast with the Mash1-negative/Dlx2-positive svz2 of the striatal anlage; shown in Figure 4G). Interestingly, the vz of the rostral-most aspect of the pallidal division (Figure 11B) lacked Dlx2 expression (Dlx2 was detected in the svz); Mash1 and Nkx2-1 expression were, however, detected (Figures 11E,H). Coronal planes caudal to the internal capsule demonstrated the shrinkage 

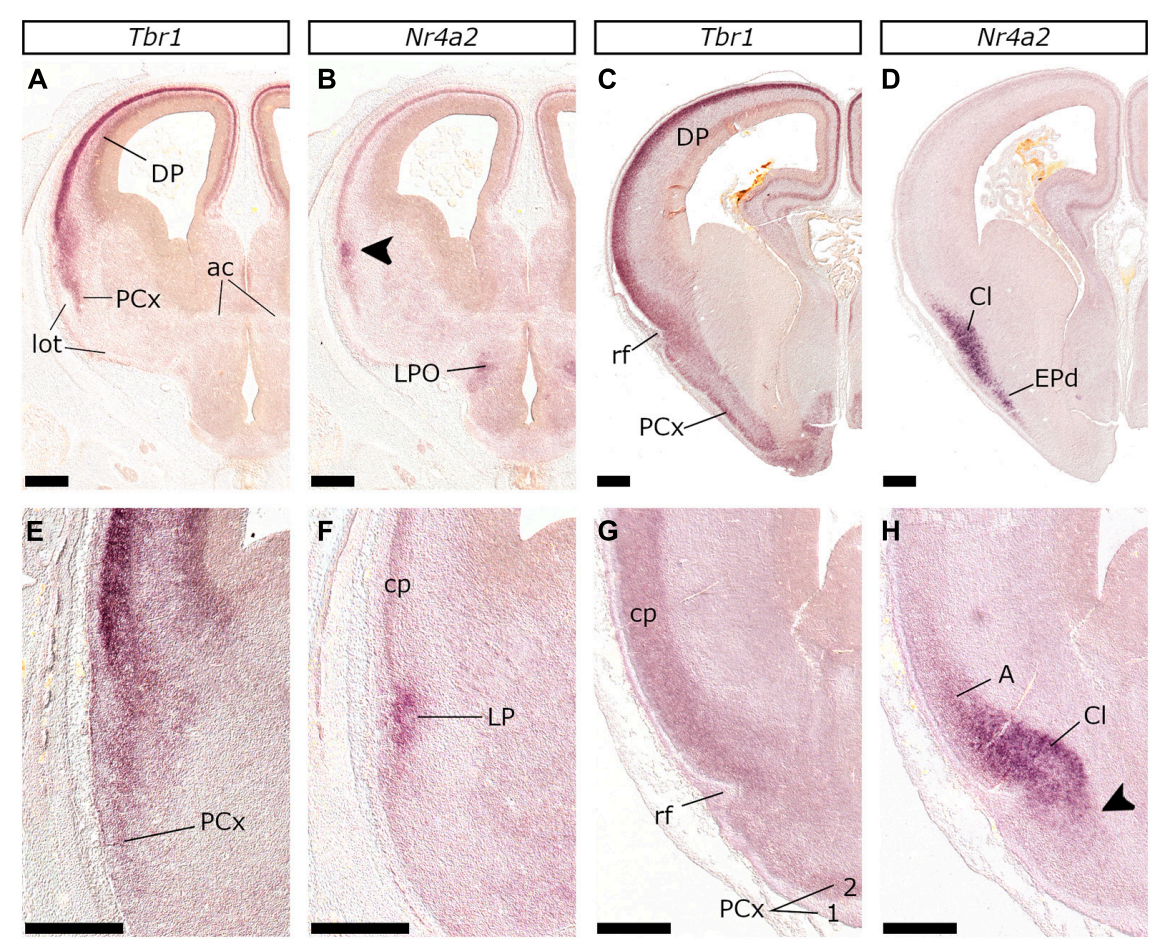

FIGURE 8 | Nr4a2 is upregulated in the LP mantle around E28/29 facilitating the delineation of the Cl primordium. In situ hybridization on coronal sections of the E28/29 (A,B,E,F) and the E34/35 (C,D,G,H) embryonic telencephalon with Tbr1 (A,C,E,G) or Nr4a2 (B,D,F,H) probes. At E28/29 Nr4a2 was detected within a subpial focus on the pallial mantle, ventral to the $\mathrm{cp}$ at the level of the ac (B) or caudal to this (F); there, Tbr1 expression became more diffuse (compared to the cp) demarcating the anlagen of the Nr4a2-expressing claustral complex and the (Nr4a2-negative) PCx primordium (A,E). At E34/35, the Cl-primordium could be visualized along the curvature introduced by the if $\mathbf{( D , H )}$. Capital letter A in $\mathbf{( H )}$ indicates migrating cells ("Arimatsu" cells) en-route to the Tbr1-expressing isocortical primordium (cp in $\mathbf{G})$. The arrowhead in $\mathbf{( H )}$ indicates the neurons of the dorsal Endopiriform Nucleus (EPd) (D) that arise in the claustrum (Cl) domain and migrate ventrally, deep to the PCx layer 2 (G). Scale bars $500 \mu \mathrm{m}$.

of the pallidal domain as shown by the expression of $D l x 2$ and Nkx2-1 (Figures 3J,J') that spatially coincided with the expansion of the CVP.

\section{The Preoptic and the Diagonal Area}

The feline POA was rostrally identified, as the wall lining the rostral-most recess of the third ventricle (Figures 12AD). Interestingly, the POA vz expressed Mash1 (Figure 12A) and Nkx2-1 (Figure 4H), lacking, however, Dlx2 expression (Figure 12B). Nevertheless, Dlx2 labeled the POA svz (and mantle), which uniquely expressed Tbr2 (Figure 12C), along with Gad2 (Figure 12D). Notably, at this level, the POA svz/mantle lacked Lhx6 expression (Figure 4F). Coronal levels around the anterior commissure further revealed that the POA (or POC, according to García-López et al., 2008) vz expressed Mash1 (Figure 12E) and Nkx2-1 (Figure 4H), but not Dlx2 (Figure 12F). At the ivf level, Er81 labeled a small part of the vz (asterisk in Figure 12H) which also expressed Mash1 (Figure 12E) but not Dlx2 (Figure 12F); this area is closely associated with the anterior commissure, in the "turn" between the evaginated and the non-evaginated telencephalic compartments (hp2 and hp1 respectively). Furthermore, a thin Er81-expressing streak of cells emanating from this region and extending into the subpallial mantle was detected (Figure $\mathbf{1 2 H}$ arrowheads). Given the transcriptional codes demonstrated in the murine telencephalon (Flames et al., 2007), previous data on POC and the Dg (GarcíaLópez et al., 2008; Puelles et al., 2016b) and the fact that Er81-lineage GP neurons arise from the latter (Nóbrega-Pereira et al., 2010) we believe that this ventricular Er81-expressing area represented the border between the Dg (dorsally) and the POA (or POC, ventrally). Lhx6 labeled the svz of the Dg and the dorsalmost domain of the POA (Figures 12G,K). Notably, the ventralmost (hypothalamus abutting) preoptic svz remained Lhx6negative (compare Figures 12F, respectively to Figures 12G,K). Furthermore, $L h \times 2$ was weakly expressed in the vz of the preoptic and the diagonal area, in contrast with the $L h \times 2$-negative pallidal anlage (Figure 12L).

\section{The Septum}

The septum encompasses the septal areal subdivisions of the striatal, pallidal, diagonal, and preoptic domains rather than being a subpallial radial domain itself (Puelles et al., 2013, 2016b). For descriptive reasons, however, we refer to the septum separately, out of the context of the radial domains. The feline striatal septum appeared in the rostral sections, anterior to the rise of the pallidal domain (Figure 11A), and, alike the central striatal division, was characterized by Dlx2, Pax6, and Er81 expression in the proliferative zones. The pallidal septal vz 


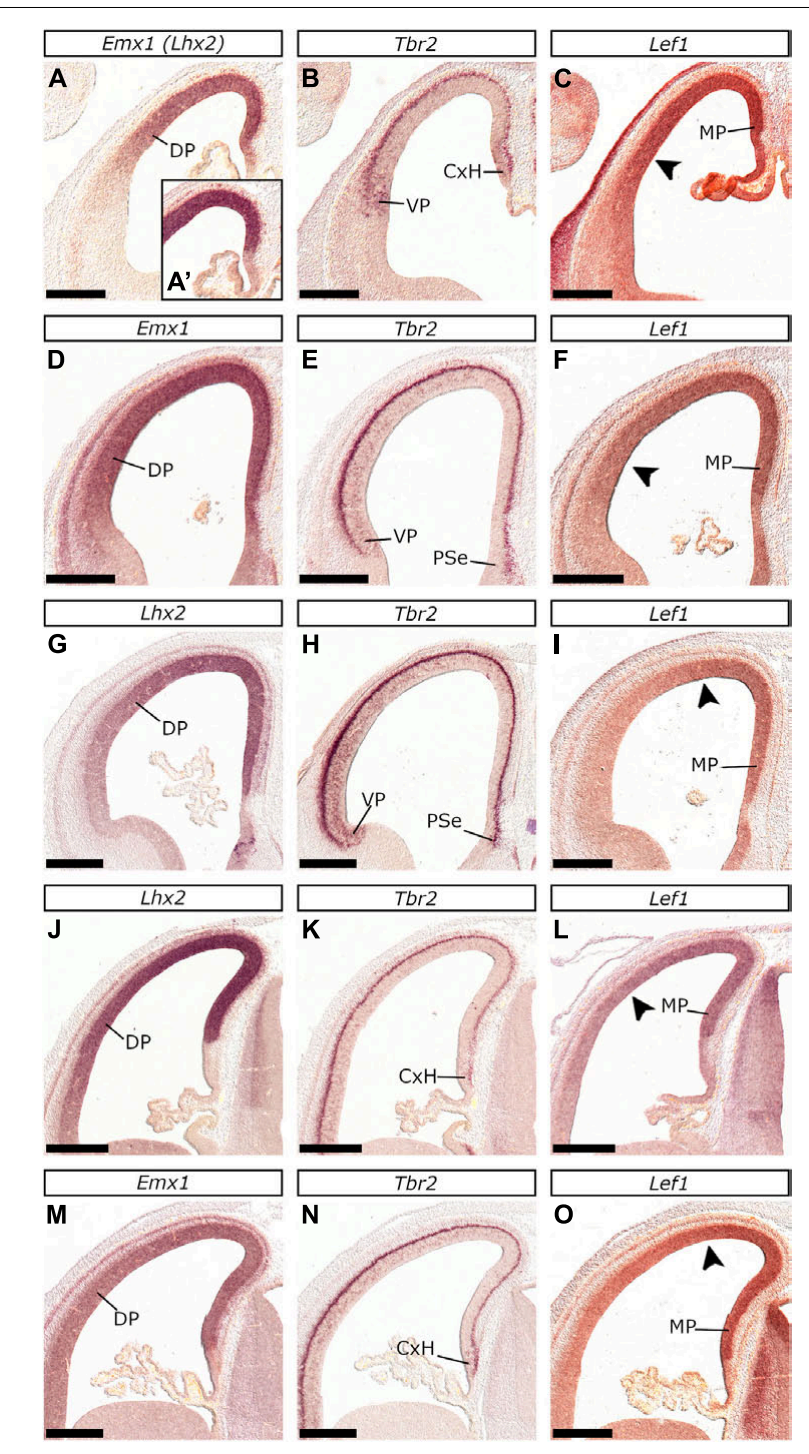

FIGURE 9 | The dynamic expression of Lef1, reveals gradual spatiotemporal restriction of the feline MP from E22/23 to E26/27. In situ hybridization on coronal sections of the feline embryonic brain at E22/23 (A-C), E24/25 (rostral: D-F; caudal: J-L) and E26/27 (rostral: G-I; caudal: M-O) with Emx1 (A,D,M), Tbr2 (B,E,H,K,N), Lef1 (C,F,I,L,O) and $L h x 2\left(\mathbf{A}^{\prime}, \mathbf{G}, \mathbf{J}\right)$ probes. The

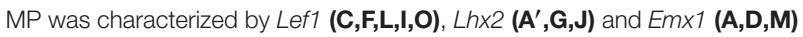
expression in the vz and Tbr2 in the svz (B,E,H,K,N). Notably, at E22/23 Lef1 was detected in a large part of the pallium (arrowhead in $\mathbf{C}$ ) almost up to the (Emx1-negative, uniquely Tbr2-expressing) vz of the VP (B). Arrowheads in (F) and (L) indicate the boundary of the Lef1 domain in the DP at E24/25, which appears restricted in comparison to E22/23 (C). At E26/27 Lef1 expression was detected in the MP and the medial-most DP sector (arrowheads in I and O). Tbr2 at E22/23 was expressed in the Vz of the VP (antihem) and the cortical hem $(\mathrm{CxH}) \mathbf{( B )}$, but at E24/25 and E26/27 its expression domain

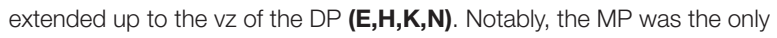
pallial sector not expressing Tbr2 in the vz up to E26/27 (H,N). Scale bars $250 \mu \mathrm{m}(\mathbf{A}-\mathbf{C})$ and $500 \mu \mathrm{m}$ (D-O).

was almost devoid of Dlx2 labeling (Figures 11B,I), however, it showed strong Nkx2-1 (Figure 11L) and Mash1 (Figure 11K) expression. The corresponding svz was Dlx2 (Figure 11I), Lhx6
(Figure 11J), and Gad2 (data not shown) positive. Notably, the pallidal molecular profile characterized the ventral half of the septal proliferative zones; the vz of the dorsal septal part (ventral to the pallial septum) was characterized by Mash1 (Figure 11K), but not $N k x 2-1$ expression (Figure 11L). The underlying svz showed high $D l \times 2$-expression (Figure 11I), being, however, $\operatorname{Lh} x 6$ negative (Figure 11J). We thus propose that the dorsal-most (pallial-septum abutting) septal part is striatal in nature. The preoptic septal subdivision is closely associated with the POA; however, apart from lack of Dlx2 expression, it did not differ molecularly from its pallidal counterpart (Figures 12A-D).

\section{The Subpallial Mantle}

The feline subpallial mantle was in general characterized by Gad2 (Figures 4K, 11P) expression. At rostral levels, Dlx2 was expressed in the subpallial septal mantle and, at low levels in the subpial territory of the paraseptal and central striatal subdivisions (arrowheads in Figures 11A,B); these subdivisions presented further a thin Pax6 expressing band below the pial surface (Figures 7F, 11F). In previous studies in mice (and chicken; Puelles et al., 2000) these territories were considered to represent the anlagen of the olfactory tubercle (OT) (adjacent to the lot) and the Acb (around the medial-ventral portion of the mantle). In the pallidal territory, strong Lhx6 expression of the Dlx2/Pax6positive subpial stream of cells (Figure 11C) was observed, with a subpopulation expressing Er81 (Figure 11D). Caudally, high Lhx6 expression was detected in the pallidal mantle; notably the subpial area of the subpallial mantle (striatal or pallidal) presented highly Lhx6 expressing cells (Figures 4F, 11J). The pallidal mantle contained the $N k x 2-1$ expressing GP anlage, that was also labeled by Gad2, $L h x 6, \operatorname{Lh} x 7$, and Er81, best demonstrated around the ivf plane (Figures $4 \mathbf{H}, \mathbf{K}, \mathbf{F}, \mathbf{J}, \mathbf{L}$ ); the expression of these markers was also observed in the compartments of the EA. Interestingly, coronal planes just rostral to the ivf and the Tbr2-expressing POA revealed a Tbr1/Pax6 expressing domain located between the Gad2-labeled central, paraseptal, and septal subdivisions of the pallidal mantle (Figures 6A,B,G,H), that we considered representing the anlagen of the DB nuclei (Puelles et al., 2000). Careful examination of E22/23 sections identified a stream of cells weakly expressing Tbr1 (arrowheads in Figure 6D) and Pax6 (arrowheads in Figure 6F) that extended between the DB and the OT, presumably reaching the VP. This was thought to represent septal-derived glutamatergic neurons en-route to the OT (Ceci et al., 2012). We propose that the Pax6/Tbr1 expressing region corresponds to the mantle of the Dg sector. Coronal planes around the level of the internal capsule showed that the Gad2 expressing subpallial mantle was surrounded by the VP, which weakly expressed Mash1 (compare Figures 110,P). Additionally, Pax6 expression in the subpallial mantle was used to identify in the cat the crescent-shaped Central amygdalar nucleus (Ce) (Figure 11R) and the anterior amygdala (AA) Figure 5A), as described in mice (Bupesh et al., 2011). Moreover, the Er81/Lhx6 expressing population represented in the cat the extended (sublenticular) amygdala (EA, Figure 11Q) as described in the mouse (GarcíaLópez et al., 2008). Caudally, the subpallial mantle reduced (compare Emx1/Dlx2 expression in Figure 3), at the expense of 

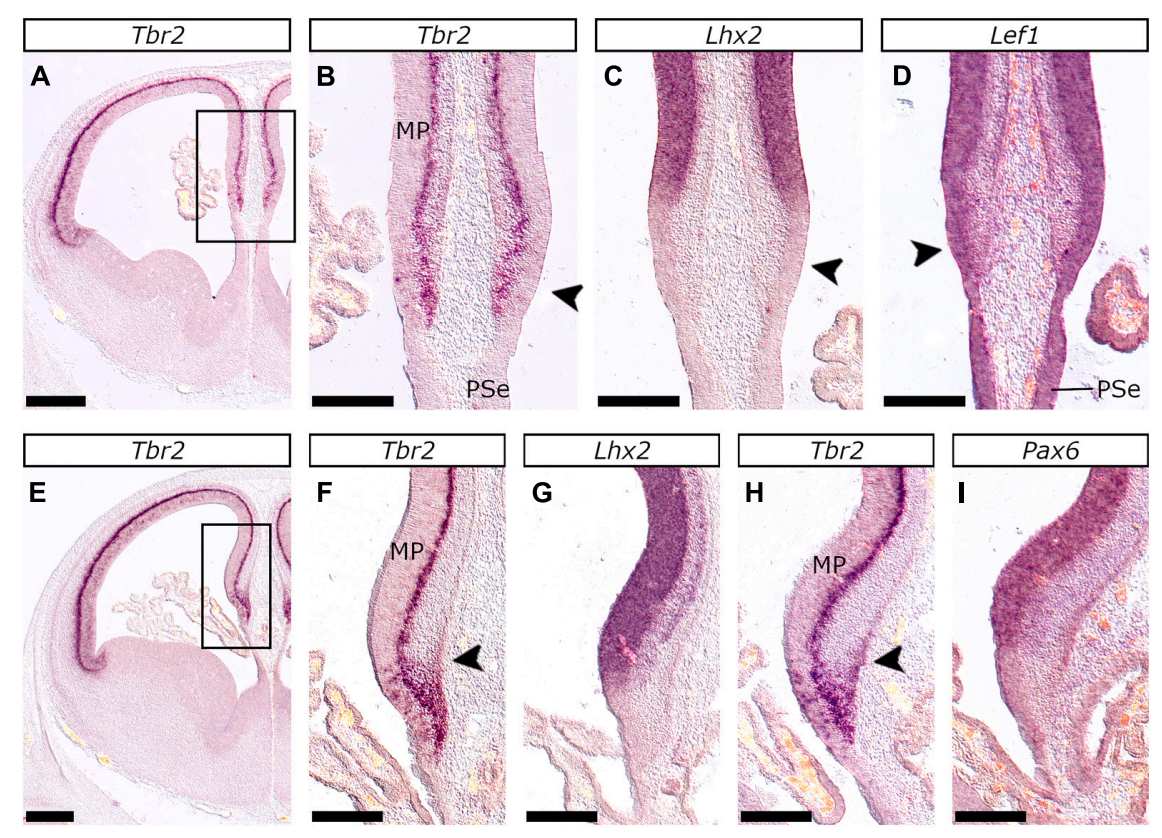

FIGURE 10 | Development of the hippocampal anlage in the E24/25 and E26/27 feline telencephalon. In situ hybridization on coronal sections of the feline embryonic pallium at E24/25 (A-D) and E26/27 (E-I) with Tbr2 (A,B,E,F,H), Lhx2 (C,G), Lef1 (D) and Pax6 (I) probes. (B-D) and (F-I) are close-ups of the boxed areas in (A) and (E) respectively. The $\mathrm{CxH}$ at E22/23 (arrowhead in B-D) was characterized by Tbr2 (B) and Lef1 (D) expression in the vz, which lacked however Lhx2 (C). Lef1 was further expressed in the PSe (D). Tbr2 was expressed in the mantle region between the CxH and the abutting MP (arrowhead in F) at E26/27; this area (arrowhead in $\mathbf{H}$ ) became wider in more advanced embryos of the same stage and represented the transient subpial neurogenic zone (or secondary matrix). Notably, the CxH did not express $L h \times 2$ (G) or Pax6 (I). Scale bars $500 \mu \mathrm{m}$ in (A,E) and $250 \mu \mathrm{m}$ in (B-D,F-I).

the pallial amygdala. As mentioned before, the caudal-most area of the basal telencephalon is molecularly ventropallial (VAP), except for its dorsal-most Dlx2/Mash1-expressing tip (AStr; Figure 3L). Notably, the Tbr2-negative core (Figure 3F) of the VAP, showed Lhx6 expression (Figure 3I), corresponding to the migrating interneurons of the caudal migratory stream (CMS) (Touzot et al., 2016).

\section{Dynamic Gene Expression in the Olfactory Bulb and the Amygdala}

The olfactory bulb and the amygdala lie at the rostral and caudal poles of the embryonic telencephalon respectively. As both the pallium and the subpallium contribute to their formation, we chose to study these entities out of the strict pallial or subpallial context. To this end, we followed the dynamic Tbr2 expression, in combination with Pax6, Tbr1, Er81, and Gad2 to approach ontogenesis of the olfactory bulb. To study the amygdala, we primarily utilized the dynamic Tbr2, Emx1, and Pax6 expression profiles, as well as $L h \times 2$ and Tbr1.

\section{The Olfactory Bulb}

The $\mathrm{OB}$ can be visualized as an evagination of the rostralmost area of the telencephalic wall, even in E22/23 embryos (Figures 13A-C). At this early stage, the $\mathrm{OB}$ consisted of two layers (like in rodents Hinds, 1968; Imamura and Greer, 2013): the Pax6-labeled ventricular zone (vz) (Figure 13B) and the Tbr2-expressing mantle (or intermediate zone, iz; Figures 13A,C). At E24/25 the vz expressed Pax6 (data not shown) similarly to E22/23; moreover, the Tbr2-labeled iz thickened (compare Figures 13B,E) and showed expression of Tbr1 (Figure 13D), Gad2 (Figure 13G), and Er81 (Figure 13F). Quite interestingly at E26/27, the OB vz, apart from Pax6 (Figure 13J), expressed high levels of Er81 (Figure 13H). At this stage, in the considerably thickened iz, Tbr2 was still expressed, yet with a distinct pattern. More specifically, two cell layers of high Tbr2 expression were detected: one demarcating the limit between the vz and the iz, and a second beneath the pia, in close contact with the olfactory nerve layer (ONL; Figure 13I and Supplementary Figures 3A, B, D). The former, further expressed Tbr1 (evident in E28/29, Supplementary Figure 3C) and was considered to represent the svz (or subependymal layer) of the OB. The subpial Tbr2-expressing stratum was evident at E28/29 and E34/35 and was considered to represent the mitral cell layer (MCL) primordium. GABAergic (Gad2-expressing) and dopaminergic (Er81-expressing) juxtaglomerular interneurons settle at the $\mathrm{OB}$ primordium around E24/25. Interestingly, within retrobulbar planes, we were able to locate strongly Er81expressing cells, migrating along the nervus terminalis, from the vomeronasal organ toward the OB anlage (Supplementary Figure 4). These were considered to represent GnRH-producing neurons that populate the basal telencephalon (Tarozzo et al., 1995; Døving and Trotier, 1998).

\section{The Amygdala}

To gain an insight into the organization of the feline pallial amygdala, we studied the dynamic Pax6, Emx1, and Tbr2 


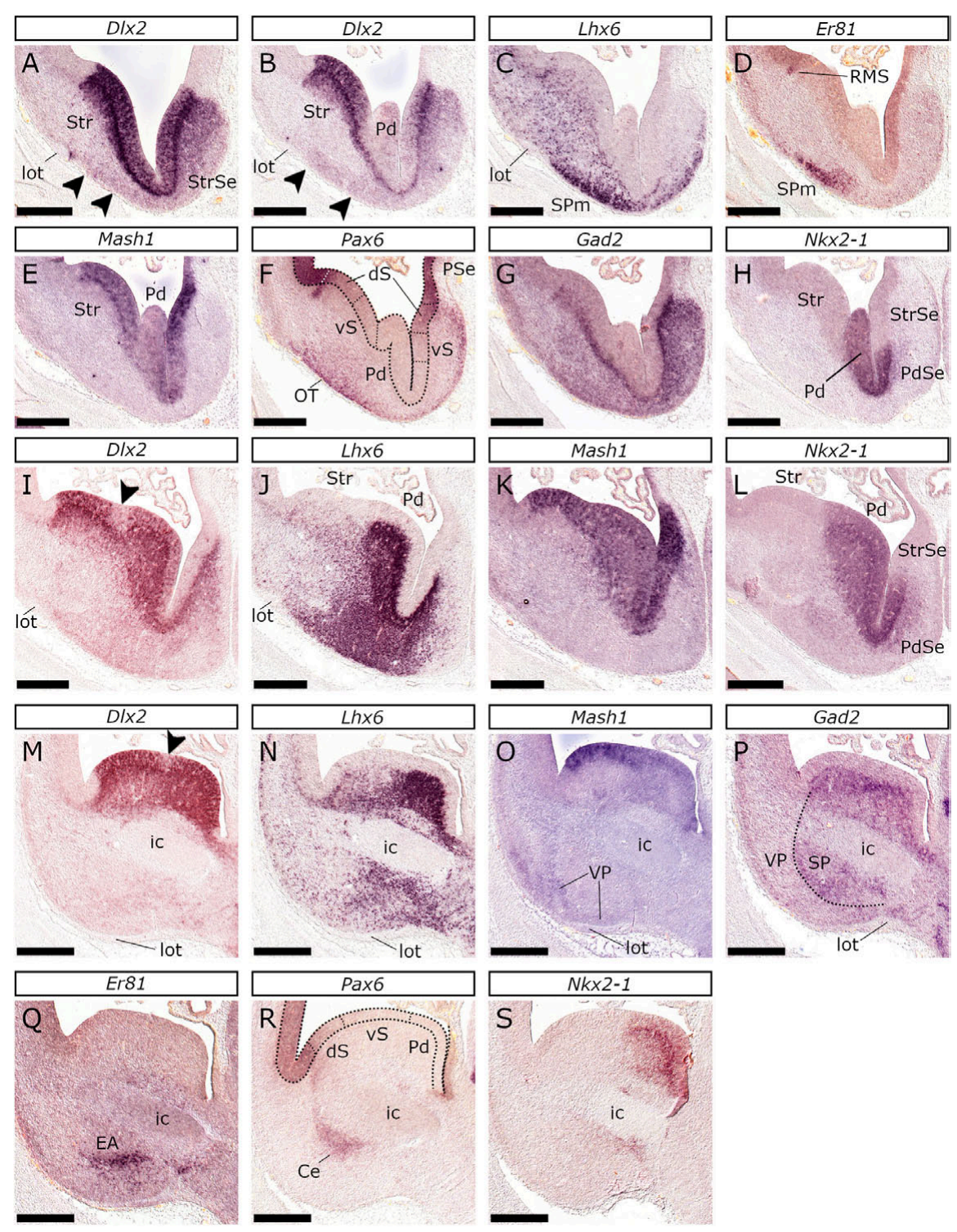

FIGURE 11 | Striatal and pallidal divisions in the subpallium of the E26/27 feline telencephalon. In situ hybridization on coronal sections of E26/27 embryonic subpallium with D/x2 (A,B,I,M), Lhx6 (C,J,N), Er81 (D,Q), Mash1 (E,K,O), Pax6 (F,R), Gad2 (G,P) or Nkx2-1 (H,L,S) probes. At rostral levels (A) D/x2 was highly expressed within the proliferative zones of the SP; DIx2 was also expressed in the mantle of the StrSe and weakly at the subpial mantle of the SP (arrowheads), up to the lot. Caudal to this level, the proliferative zones of the SP expressed D/x2 (B), Mash1 (C), and Gad2 (G), while Nkx2-1 (H) delineated the Pd. The proliferative zones of the Str were divided into a dorsal (dS) and a ventral (vS) part; Pax6 (F,R) and Er81 (D) were expressed in the former. In the svz of the dS, a small population expressing Er81 and Pax6 represented the primordium of the RMS (D,F). Nkx2-1 (H,L) and Pax6 (F) expression divided the septum in Pd (PdSe), ventral striatal (vS) and dorsal striatal (dS) subdivisions. The mantle of the central subdivision of the SP expressed $L h \times 6$ (C) and Er81 (D). Pax6 expressed subpially, demarcated the OT anlage (F). Note that the vz of the rostral aspect of the Pd did not express D/x2 (B). The Pd svz was characterized by DIx2 (I,M), Mash1 (K,O), Nkx2-1 (L,S) and Lhx6 (J,N) expression; in contrast, the Str svz expressed only D/x2 (I,M). D/x2 was not detected in the region between the central subdivisions of the Str and the Pd (arrowhead in I,M) that corresponds to the pLGE4-pMGE1 regions. At levels around the ic (M-S) weak expression of Mash1 was observed in the VP along the lot (O). The dotted line in (P) indicates the PSB. Pax6 was expressed in the crescent-shaped Ce primordium (R), while Er81 demarcated cell populations of the EA (Q) related to the GP ("sublenticular"). Scale bars $500 \mu \mathrm{m}$.

expression patterns at E22/23, E24/25, and E26/27, using not only coronal but also sagittal and horizontal sections. At E22/23, diffuse Emx1 expression (Figures 14D,H,I) was detected within the VAP mantle (VAPm). Lhx2 further labeled the VAPm (Figures 14C,G), which also expressed Tbr2 (Figures 14B,F) and Pax6 (Figures 14A,E) in a "salt-and-pepper" pattern. At E24/25,
Pax6, Emx1 and Tbr2 expression profiles within the VAPm changed from diffuse to focal. More specifically around the level of the PThE, Pax6, Emx1, and Tbr2 expressing cells gathered to form foci, positioned between the Ce primordium and the vz of the VAP (Figures 14J,K). At horizontal levels ventral to this, Pax6 expression was reduced (Figure 14N) and Emx1 labeled 

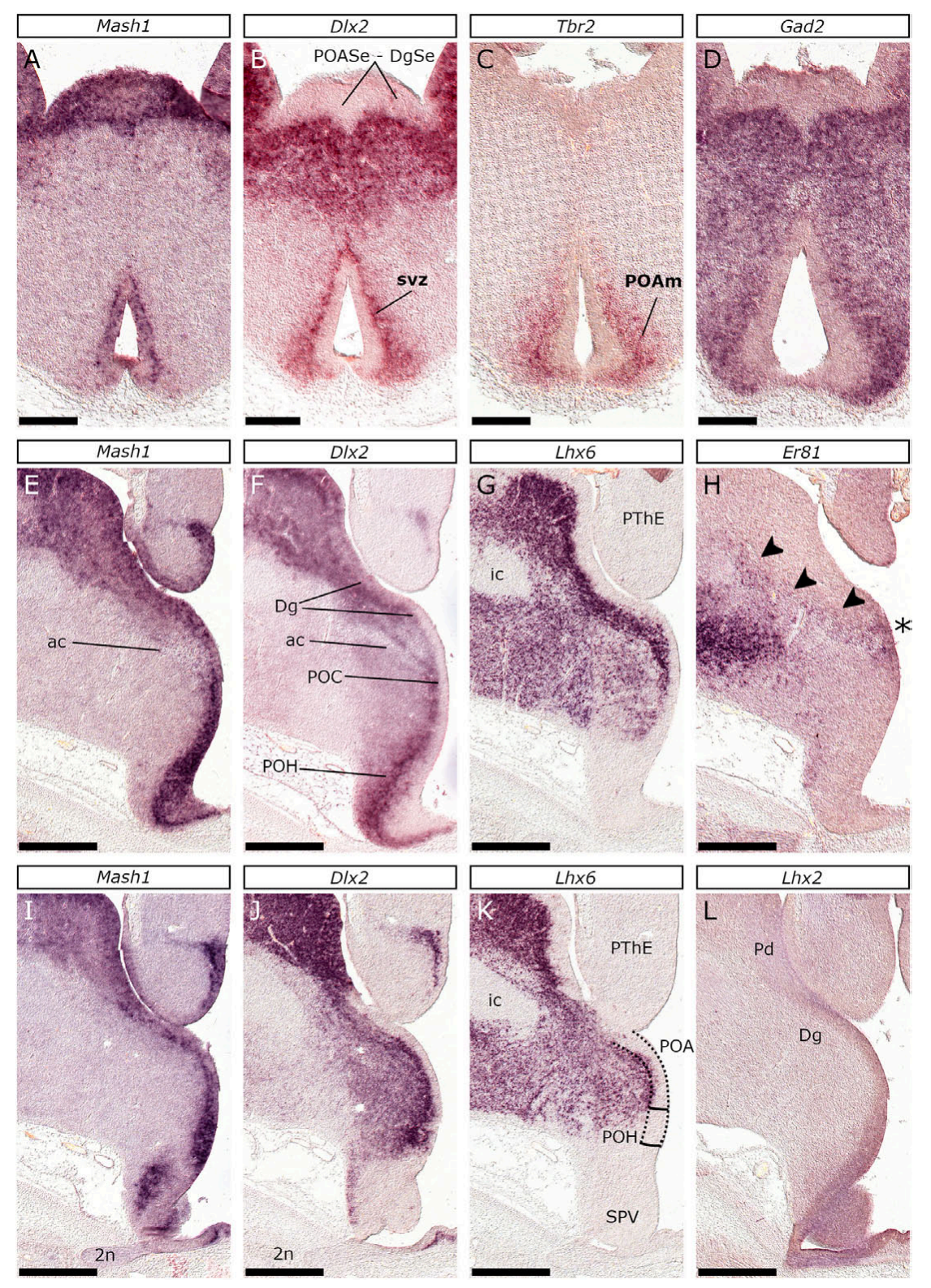

FIGURE 12 | The diagonal and the preoptic area at E26/27 are characterized by a lack of $D / x 2$ expression in the vz. In situ hybridization on coronal sections of the E26/27 embryonic subpallium with Mash1 (A,E,I), D/x2 (B,F,J), Tbr2 (C), Gad2 (D), Lhx6 (G,K), Er81 (H) or Lhx2 (L) probes. The POA was characterized by Mash1 expression in the VZ (A,E,I) and lack of DIx2 (B,F,J) which was nevertheless expressed in the POA svz (svz in B); Tbr2 (C), and Gad2 (D) were detected in the POA mantle (POAm in $\mathbf{C})$. Lhx6 was expressed in the dorsal-most area of the POA (G,K), which is associated with the ac (POC in $\mathbf{F})$. Notably, Lhx6 expression reduced at the hypothalamus-abutting POA (POH). Lhx2 was detected within the vz of the POA and the Dg (L); around this level, Er81 expression was detected in the vz between the Dg and the POA (asterisk in $\mathbf{H}$ ). Arrowheads in $\mathbf{( H )}$ indicate neurons migrating from the Dg to the GP. Scale bars $250 \mu \mathrm{m}(\mathbf{A}-\mathbf{D})$ or $500 \mu \mathrm{m}(\mathbf{E}-\mathbf{L})$.

cells (Figure 140) gathered more superficially, near the lot. Tbr2 expressing cells (Figure 14P) on the other hand, were detected dispersed, more medially, with a pattern almost complementary to that of Emxl (compare Figures 140,P). In sagittal sections (Figures 14M,Q), the Tbr2 expressing domain could be divided into a dorsal/deep domain, in close association with the vz of the VAP, and a ventral territory extending superficially; Tbr2 expressing cells appeared tightly assembled in the former and dispersed in the latter (Figure 14Q).
At E26/27, coronal sections at levels caudal to the ic revealed diffuse Pax6 expression (Figure 5A) in the transition region between the caudal Ce and the AA. Weak Lhx2 expression could be further observed in a cell population emanating from the VP and extending deep to the AA (Figure 5C). At this plane, Tbr2 expression was observed in a small superficial part of the VAPm that also showed Pax6 expression (compare Figures 5B,C). Tbrl expression extended all along the lot up to the bed nucleus of the accessory olfactory tract (BAOT) 

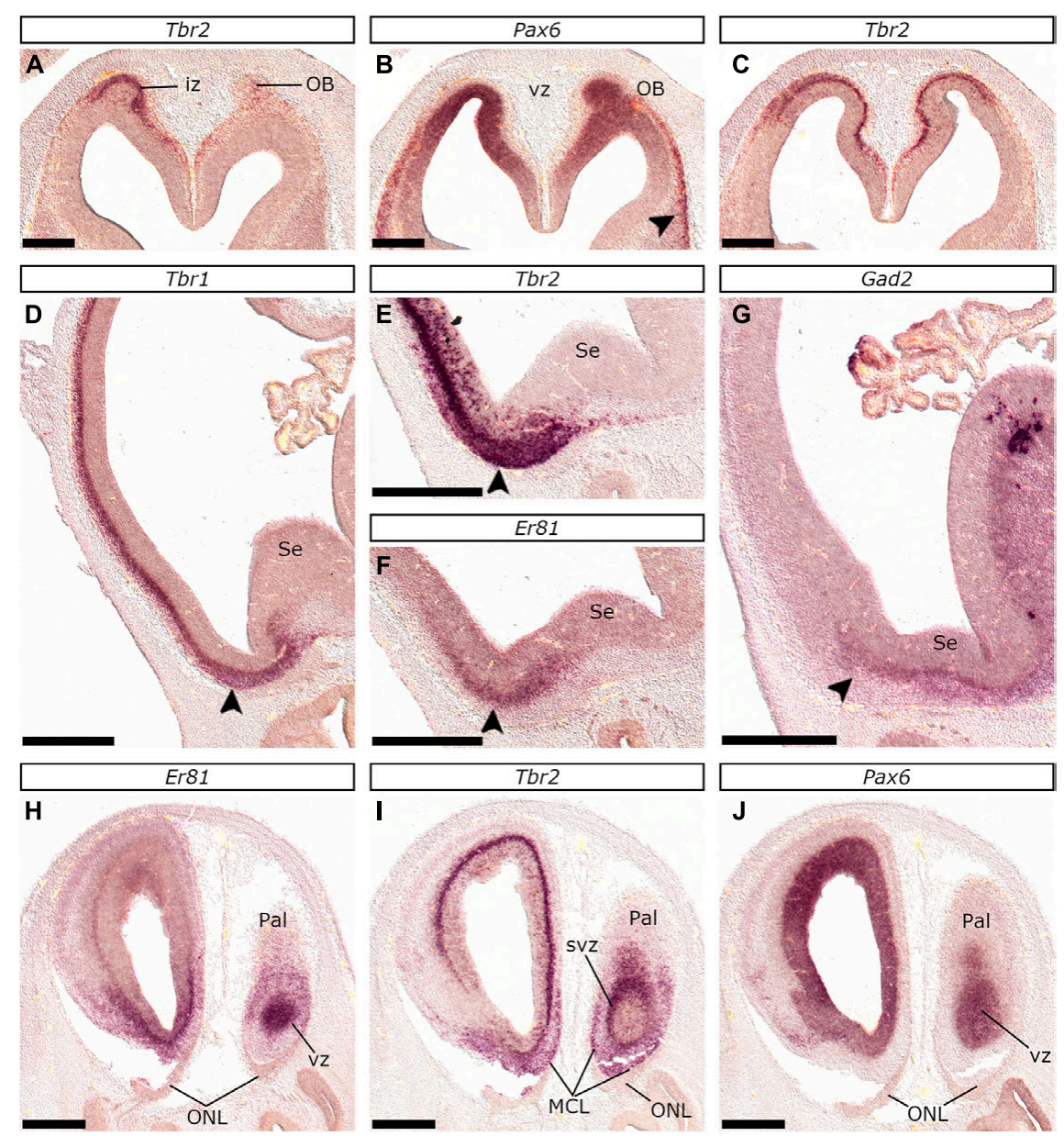

FIGURE 13 | Development and neurogenesis in the feline OB between E22/23 and E26/27. In situ hybridization on coronal sections at E22/23 (A-C) and E26/27 (H-J), or sagittal sections at E24/25 (D-G) of embryonic brains with Tbr2 (A,C,E,I), Pax6 (B,J), Tbr1 (D), Er81 (F,H), or Gad2 (G) probes. At E22/23, the OB could be identified as an evagination of the rostral-most pallial region, that expressed Pax6 in the vz (B) and Tbr2 in the iz (A,C). Arrowhead in (B) points to Pax6-expressing neurons of the olfactory cortex. Sagittal sections at E24/25 revealed considerable thickening of the iz, which highly expressed Tbr1 (D) and Tbr2 (E). Er81 (F) and Gad2 (G) were expressed in the OB primordium indicating settlement of SP-derived interneurons. Arrowheads in (D-G) indicate the OB primordium. Coronal sections at E26/27 showed that the vz of the OB expressed Pax6 (J) along with Er81 (H). Furthermore, the iz was considerably thickened, while Tbr2 expression was restricted in the basal aspect of the vz (svz in I) and the subpial area; the latter was in close contact with the ONL and represented the incipient MCL. Scale bars $250 \mu \mathrm{m}$ (A-C) or $500 \mu \mathrm{m}$ (D-J).

primordium (Figure 5D). Caudal to this level, almost identical Pax6 and Tbr2 expression patterns were observed, with a dorsal and a ventral domain (Figures 5E,F) surrounding the Emx1expressing territory (Figure 5G). Given that Emx1 is expressed in the anlagen of the BL and the PLCo (Puelles et al., 2000; Medina et al., 2004), whereas Pax6 within the BM (Puelles et al., 2000) and the Me (Bupesh et al., 2011; Duan et al., 2013), we identified the dorsal Pax6/Tbr2 expressing focus as the BM anlage. Moreover, we considered the superficial Tbr2-expressing domain to represent superficial corticoid nuclei (ACo), given (1) its position between the Emx1 positive BL/PLCo and the Emx1/Tbr1 negative Me primordia and (2) the fact that both BMA and ACo are products of the anterior amygdalar radial unit (Garcia-Calero and Puelles, 2021). On the other hand, it could belong to the MePV, given that its core has been reported to express both Pax6 and Tbr2 (Ruiz-Reig et al., 2018). Careful examination of Lhx6 preparations at the same coronal level identified the NLOT, as a focus almost devoid of subpallialderived migrating interneurons (Figure 3G and schema). At levels immediately posterior to the previous, the BM anlage (Tbr2/Pax6-expressing, dorsal domain) continued to extend caudally (Figures 5I,J), while the rest of the VAPm expressed Emx1 (data not shown) and Tbr1 (Figure 5L). Finally, at the caudal-most planes of the VAPm, neither Tbr2 (Figure 3E) nor Pax6 (data not shown) expression was detected.

\section{DISCUSSION}

Comparative gene expression analysis and molecular fate mapping have revealed the principles underlying vertebrate brain design, leading to the emergence of the field of evolutionary 


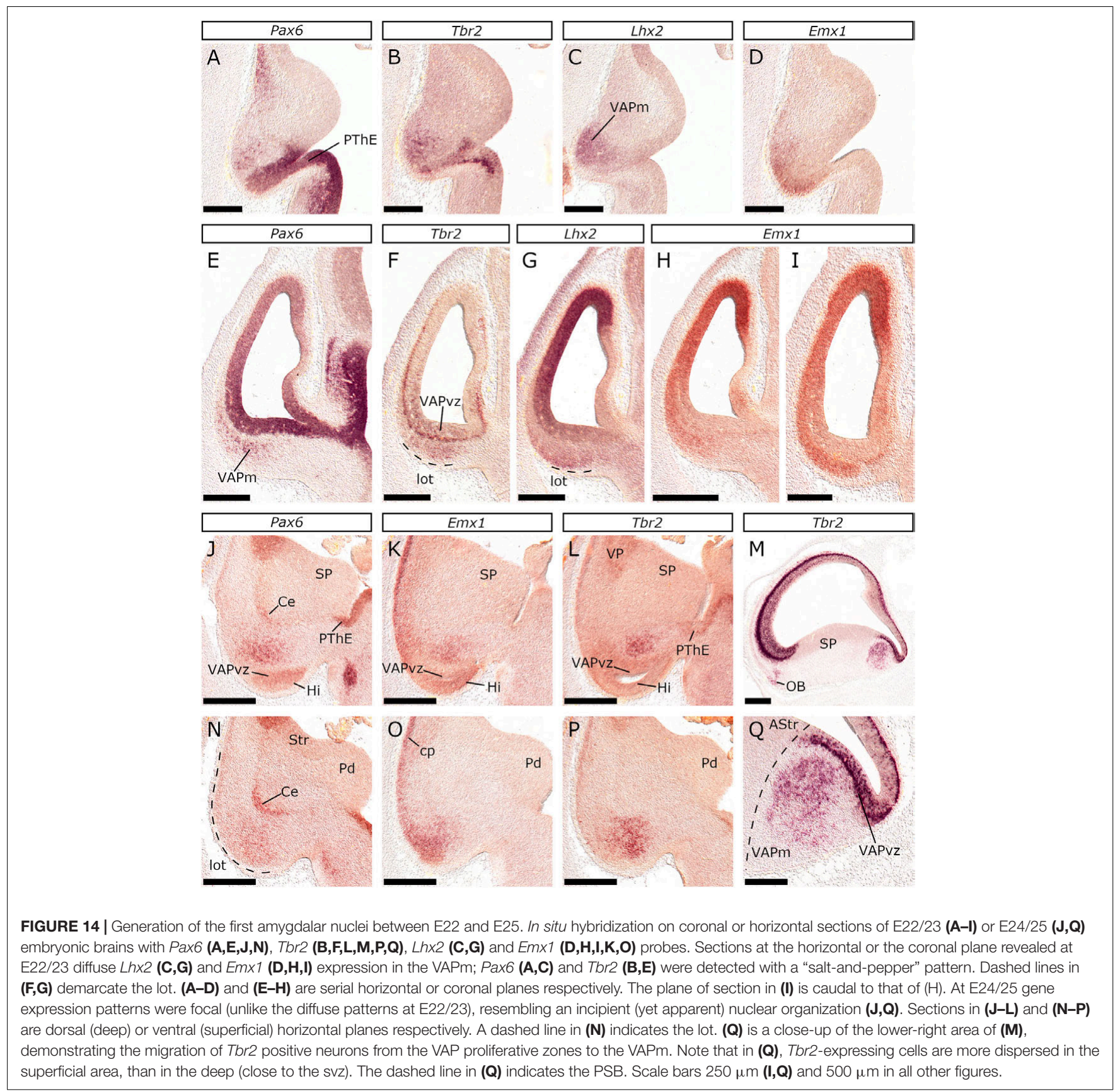

developmental neurobiology (neuro-evo-devo, Striedter, 2007). Gene expression studies, however, not only unravel brain genoarchitecture, described by the prosomeric model and the relevant ontology, but also pinpoint differences, that may affect the brain morphology and function even within a small taxonomic group, like mammals (Molnár et al., 2006; Rakic, 2009; Martínez-Cerdeño et al., 2018). In this work, we have analyzed the genoarchitectonic compartmentalization of the developing feline telencephalon, studying the expression of genes, the orthologs of which have been successfully used in other vertebrates (e.g., Puelles et al., 2000; Flames et al., 2007; Abellán et al., 2014; Desfilis et al., 2018).
In the developing feline pallium, the expression of Pax6, Tbr2, and Tbr1 marks radial glia, intermediate progenitors, and post-mitotic neurons, or $\mathrm{vz} \rightarrow \mathrm{svz}$ (already present at E22/23) $\rightarrow$ mantle, respectively. Additionally, neurogenesis is spatiotemporally regulated: the VP mantle is the first to appear, while the MP mantle is the last; as in mice, a gradient of Tbr1 expression with high levels in the VP mantle progressively decreasing toward the MP mantle was observed (Bulfone et al., 1995; Medina et al., 2004). The pallial mantle appeared at E22/23 as Tbr1 expressing cells aggregate forming a thin lamina; the cp however was clearly detected one day later at E24/25 as a Tbr1-expressing layer. At this stage, lamination within the pallial 
mantle, correlated with the Tbr1 expression in the cp and the (Tbr1-positive) subplate (sp)/intermediate zone (iz); these are clearly detected as distinct layers in the lateral telencephalic wall and merge gradually to form a single layer in the MP. In addition, the DP cp is clearly separated from the underlying sp/iz at E24/25, while the MP cp at E28/29 (Figure 8A). The dynamic expression pattern of Tbr2 in the vz further supports a sequential mode of pallial neurogenesis. In mice, Tbr2 upregulation in the vz reflects an increase in the production of intermediate progenitors at the peak of neurogenesis. Interestingly, in the feline embryo, at E22/23, the only pallial domain expressing Tbr2 is the VP vz. At E24/25 and E26/27 however, Tbr2 expression domain gradually expands into the vz of the DP. Accordingly, the vz of the MP is the last to upregulate Tbr2 expression around E30. Previous research in cats has demonstrated that subplate cells appear around E24, while layer VI neurons of the visual cortex later, at E31 (Luskin and Shatz, 1985a,b). Moreover, in a recent study (Glatzle et al., 2017) using Tbr1 as a marker, E30 was identified as the stage when deep-layer neurogenesis in cats commences. These results, however, are not in discrepancy with our study, given the heterochrony in the generation of the $\mathrm{cp}$ across the various pallial sectors. Indeed, the primary visual cortex (Luskin and Shatz, 1985a,b), along with the dorsal-lateral telencephalic region that was studied by Glatzle et al. (2017), are considered dorsal pallial derivatives; it remains also unclear if earlier stages were screened in the latter study. In mice, Cajal-Retzius (CR) neurons are generated between E10.5 and E12.5 and express, among other factors, Tbr1 (Hevner et al., 2003; Yoshida et al., 2006; Tissir et al., 2009). We show that in the feline pallium CR neurons first appear as a Tbr1-expressing subpial stream, in the basal telencephalon of E22/23 embryos. We also show that CR neurons also express Tbr2 (Figure 7A). Interestingly at rostral levels, Tbr2 is also highly expressed in the mantle of the septum and the VP - both regions are major sources for $\mathrm{CR}$ neurons in mice (Bielle et al., 2005). As of E24/25, subpial expression of Tbr1 and Tbr2 in the basal telencephalon gradually decreases, suggesting that CR generation peaks around E22/23.

At E26/27 the proliferative zones of the VP formed a C-shaped ring, an observation following the concentric ring pallial model (Puelles et al., 2019). The identification of the vz and the svz of the VP was primarily based on the analysis of Tbr2, Pax6, and Emx1 expression. Previous studies in mice have shown that the VP vz and svz express Dbx1, Sfrp2, Fgf15, Lhx9, and Gdf10 (Puelles et al., 2000; Kim et al., 2001; Medina et al., 2004; GarcíaLópez et al., 2008; Ruiz-Reig et al., 2018); these, however, are detected within distinct subregions of the VP, hence, we did not use them in this study. Interestingly, Emx1 is not expressed in the $\mathrm{vz}$ of the VP; this observation led to the updating of the pallial model from tripartite to tetrapartite (Smith-Fernandez et al., 1998; Puelles et al., 2000). Pax6 is strongly expressed in the $\mathrm{vz}$ of the VP (considered to be the antihem), regulating the expression of a battery of genes essential for the formation of the PSB (Kim et al., 2001; Stenman et al., 2003b; Tuoc and Stoykova, 2008; Carney et al., 2009). Lhx2 on the other hand, antagonizes Pax6, suppressing the antihem fate (Godbole et al., 2017); Lhx2 is, thus, weakly expressed in the vz of the VP. Our results from the analysis of these genes were in accordance with previous findings in mice. Therefore, based on the above, as well as in the observation that Tbr2 is expressed in the antihem as early as E22/23, we mapped the proliferative zones of the VP (Schemata in Figures 2-4): (1) In the retrobulbar septal region; (2) In the lateral-ventral telencephalic wall; (3) In the caudal-most aspect of the basal telencephalon (vz of the VAP); (4) Continuous with the PThE, in the medial aspect of the basal telencephalic bulge (CVP).

In the updated prosomeric model, the LP has been redefined to include the claustroinsular complex that exclusively expresses Nr4a2 (Puelles, 2014; Puelles et al., 2016a; Watson and Puelles, 2017). Our results show that in the domestic cat, Nr4a2 is expressed in the mantle of the LP after E28/29. Notably, this territory is located subpially between the Tbr1-expressing $\mathrm{cp}$ (dorsally) and the PCx primordium that is more diffusely labeled by Tbr1 (ventrally). Around E34/35, Nr4a2 expression marked, apart from the claustroinsular primordium, the migration route of Cl-derived cells toward the isocortex ("Arimatsu" cells) or the EPd (Arimatsu et al., 2009; Puelles, 2014). We did not detect Nr4a2 in LP at earlier stages, however, we observed expression of Tbr 2 and Pax6 in the LP mantle at the ventral-most border of the Tbr1-expressing $\mathrm{cp}$ in rostral (yet retrobulbar) pallial fields of E22/23 and E24/25 embryos (rld in Figure 7). This domain could represent the $\mathrm{Cl}$ primordium; however, this hypothesis cannot be supported as we did not detect Tbr2/Pax6 expression at caudal levels.

In mice, the MP is strongly associated with the expression of the Wnt effector, Lef1 (Behrens et al., 1996; Galceran et al., 2000). We also observed a dynamic expression pattern of the feline Lef1 ortholog. At E22/23, the MP primordium covers a large part of the pallium, in later stages, however, DP develops and MP occupies a relatively smaller part. More specifically, at E22/23 Lef1 expression domain extends throughout the pallial anlage, except for the vz of the VP (antihem) that expresses Pax6 known to downregulate Wnt signaling (Chou and Tole, 2019). At later stages, however (E24/25 - E26/27), the Lef1 expression domain is gradually restricted to the vz of the MP and the MPabutting DP. This dynamic pattern of Lef1 is also evident in the developing mouse pallium (Abellán et al., 2014, Allen Brain database). Given that MP is the hippocampal primordium, we further propose that, in terms of hippocampal development, the E26/27 feline MP corresponds to the E14.5 murine MP. Even at E22/23, the vz and the svz of the feline $\mathrm{CxH}$ expressed Tbr2, as already described for mice (Sugiyama et al., 2013). At E26/27, the medial-most ( $\mathrm{CxH}$-abutting) mantle of the MP thickened, while in the pial surface and the "mantle" corresponding to the $\mathrm{CxH}$, a $T b r 2$ population was detected that appeared expanded in older embryos within the same group. This population may represent Tbr2-expressing CR cells of the transient subpial neurogenic zone (or hippocampal secondary matrix) identified at E14.5 in mice (Li et al., 2009; Hodge et al., 2013; Urbán and Guillemot, 2014).

Our study shows that subpallial organization is conserved at least between cats, mice (Flames et al., 2007; García-López et al., 2008) and chicken (Abellán and Medina, 2009; Bardet et al., 2010). This was revealed by the analysis of the expression profiles of genes that delineate striatal, pallidal, diagonal, and preoptic divisions. The striatal domain is shown to parcellate in a dorsal and a ventral compartment, identified by Pax6 and Er81 within 
the $\mathrm{vz}$ of the former. The dorsal striatum is, in rostral levels, further characterized by focal expression of Pax6 and Er81 in the svz. This domain was considered to represent the rostral migratory stream primordium supplying the OB with $\mathrm{Pax6/Er81}$ -expressing periglomerular neurons. This cell population has been shown to express Er81 in mice (Stenman et al., 2003a; Long et al., 2007; Figure 1A of Carney et al., 2009; Figure 4A of Rash et al., 2013). Additionally Flames et al. (2007), defined the pLGE1 as the vz region overlying the svz Er81 focus (see in Flames et al., 2007 their Figure 2D). On the other hand, the same authors identified this focal Pax6 expressing domain dorsal to pLGE1 (Flames et al., 2007 their Figure 2B) ascribing it to the pallium. Having studied Er81 and Pax6 on consecutive sections along the rostrocaudal axis we propose that it is the same population that expresses Er81 and Pax6.

Previous studies in mice have shown that at E12.5 and E13.5, a small vz domain located at the border between the ventral Str (pLGE4) and the dorsal Pd (pMGE1), lacks the expression of Dlx2 but expresses Nkx6-2 (Flames et al., 2007). In our experiments, the vz of the rostral-most region of the Pd shows high Nkx2-1 and Mash1 expression but lacks Dlx2; this indicates that pMGE1 expands to this rostral area. We did not detect Lhx6 or $\operatorname{Lh} x 7$ expression in the $\mathrm{vz}$ of the $\mathrm{Pd}$; thus, we were not able to delineate the rest of the $\mathrm{Pd}$ progenitor domains described previously in mice (Flames et al., 2007). The POA in felids can be divided into a dorsal area that is associated with the ac, and a ventral that abuts the hypothalamus, as already described for mice (Abellán et al., 2010). An interesting observation is the identification of a subset of Tbr2 expressing cells within the Gad2-expressing POA mantle. These cells may relate to the POA-native expression of pallial markers $D b x 1$ or Lhx5 (Flames et al., 2007; Gelman et al., 2009; Abellán et al., 2010), or may have migrated from extratelencephalic regions, like the PThE or the telencephalon-opto-hypothalamic domain (Morales et al., 2021). This observation reflects the vast diversity of cellular phenotypes (e.g., cholinergic, GABAergic, glutamatergic, nitrergic or even dual-phenotype Glu-GABAergic neurons, as well as oligodendrocytes) produced by the POA (Kocsis et al., 2003; Ottem et al., 2004; Abellán et al., 2010; Medina and Abellán, 2012). Furthermore, our results suggest that the POA proliferative zones include a subventricular zone, although its existence in mice has been disputed (Gelman et al., 2009). This hypothesis is supported by the detection of a Dlx2expressing streak of cells forming a line abutting the basal aspect of the POA vz (Mash1-expressing) and the POA mantle (Gad2expressing).

In cats, the primordium of the $\mathrm{OB}$ was evident at E22/23, as an evagination of the rostral-most telencephalic domain; at this stage, Pax6 and Tbr2 labeled the vz and the iz, respectively. In mice evagination does not commence prior to E12 (Hinds, 1968); the OB anlage expresses Pax6, Tbr2, and Tbr1 as of E11 (Imamura and Greer, 2013). Given that the first mitral cells in mice are generated via the non-canonical (Pax6 $\rightarrow$ Tbr $1 \rightarrow$ Tbr2) neurogenic cascade (Imamura and Greer, 2013), we studied Tbr2 at E22/23, instead of Tbr1, to label the nascent projection neurons. Tbr1 expression was nevertheless detected at E24/25, marking the whole extent of the iz. At E26/27, the nascent MCL appears at the ventral-lateral aspect of the $\mathrm{OB}$ as a thin subpial layer expressing Tbr2, in close contact with the ONL.

Although in mice the svz of the OB does not express Tbr2 (Imamura and Greer, 2013), we observed a Tbr2 positive layer lining the basal aspect of the $\mathrm{OB} v \mathrm{vz}$ at E26/27 and later stages. This temporally coincided with the thickening of the iz and was thought to represent neurogenesis of projection neurons through the canonical cascade (Winpenny et al., 2011). Given that the emergence of the svz has been proposed to associate with the ability to better control neurogenesis and to overcome the limitation of the $\mathrm{vz}$ in terms of neuronal output (ventricular choke hypothesis, Martínez-Cerdeño et al., 2016), we propose the existence of $\mathrm{Tbr}$-expressing intermediate progenitors in the $\mathrm{OB}$ of cats as of E26/27.

Interneurons settle in the feline $\mathrm{OB}$ around E24/25, as indicated by the upregulation of Gad2 and Er81 expression between the $\mathrm{vz}$ and the $\mathrm{iz}$ of the $\mathrm{OB}$. In mice, most $\mathrm{OB}$ interneurons are generated after E12.5 (Díaz-Guerra et al., 2013) by dorsal striatal progenitors regulated by $D l x 2$ and Mash 1 among others (Allen et al., 2007; Long et al., 2007). As mentioned above, we focused on Er81, which is crucial for the development of the dopaminergic interneurons (Cave et al., 2010), and Gad2 that has been linked to periglomerular neurons (Esclapez et al., 1993, 1994). Our results further suggest that the OB may be capable of producing its own interneurons after E26/27; this conclusion is based on the high Er81 expression detected within the vz, as in mice (Stenman et al., 2003a; Long et al., 2007).

While studying the genoarchitecture, we also defined the timing of several events and showed that staging equivalence should be studied concerning the process. Our results suggest a complex pattern of heterochronies between the mouse and the cat; represented by shifts in the timing of events (some events occurring earlier in the cat or vice versa). For instance, E22/23 is generally equivalent to the E11.5 stage of the mouse: olfactory bulb evagination, however, in the cat has already commenced at E22/23, while in mice this event takes place after E12 (Hinds, 1968; Imamura and Greer, 2013). On the other hand, the murine LP expresses Nr4a2 as of E12.5 (Puelles, 2014), this upregulation in the feline LP is observed much later, after E28/29 (approximately a week later after the murine equivalent stage). Moreover, as expected, in the feline telencephalon developmental processes usually take much longer to complete; this may be used to accurately define distinct stages of a developmental process.

In summary, our results show that the overall genoarchitecture of the cat telencephalon is conserved, with territories topologically equivalent to those previously described for the mouse and other vertebrate species, following the prosomeric model and the derived ontology (Puelles et al., 2000, 2013, 2016a; Brox et al., 2004; Abellán and Medina, 2009; Abellán et al., 2014; Desfilis et al., 2018). Furthermore, they underline the heterochronous nature of developmental events in a comparative context between species. These results, along with the fact that the feline brain is gyrencephalic, suggest that cats can provide a useful animal model in the study of the brain in ontogenesis and evolution. To our knowledge, this is the first systematic analysis of the feline telencephalon genoarchitecture and we hope that it will provide a reference for future research, especially 
given the re-appearance of this species in neuroscience research (Hinova-Palova et al., 2019; Valdés-Cruz et al., 2019; Graff et al., 2020).

\section{DATA AVAILABILITY STATEMENT}

The original contributions presented in the study are included in the article/Supplementary Material, further inquiries can be directed to the corresponding author.

\section{ETHICS STATEMENT}

Ethical review and approval was not required for the animal study because the feline embryos or fetuses were obtained from domestic cats referred to the Unit of Obstetrics and Surgery of the Companion Animal Clinic of the School of Veterinary Medicine, Faculty of Health Sciences, Aristotle University of Thessaloniki for preventive ovariohysterectomy. In case of pregnancy, the excised gravid uterus was not processed for incineration, but immediately incised - this process does not require approval.

\section{AUTHOR CONTRIBUTIONS}

NS conceptualized and designed the study, performed most of the experiments, and wrote the first draft of the manuscript. CV performed the ovariohysterectomies, the

\section{REFERENCES}

Abellán, A., and Medina, L. (2009). Subdivisions and derivatives of the chicken subpallium based on expression of LIM and other regulatory genes and markers of neuron subpopulations during development. J. Comp. Neurol. 515, 465-501. doi: $10.1002 /$ cne. 22083

Abellán, A., Desfilis, E., and Medina, L. (2014). Combinatorial expression of Lef1, Lhx2, Lhx5, Lhx9, Lmo3, Lmo4, and Prox1 helps to identify comparable subdivisions in the developing hippocampal formation of mouse and chicken. Front. Neuroanat. 8:59. doi: 10.3389/fnana.2014.00059

Abellán, A., Vernier, B., Rétaux, S., and Medina, L. (2010). Similarities and differences in the forebrain expression of Lhx 1 and Lhx 5 between chicken and mouse: insights for understanding telencephalic development and evolution. J. Comp. Neurol. 518, 3512-3528. doi: 10.1002/cne.22410

Allen, Z. J., Waclaw, R. R., Colbert, M. C., and Campbell, K. (2007). Molecular identity of olfactory bulb interneurons: transcriptional codes of periglomerular neuron subtypes. J. Mol. Histol. 38, 517-525. doi: 10.1007/s10735-007-9115-4

Arimatsu, Y., Nihonmatsu, I., and Hatanaka, Y. (2009). Localization of latexin-immunoreactive neurons in the adult cat cerebral cortex and claustrum/endopiriform formation. Neuroscience 162, 1398-1410. doi: 10.1016/ j.neuroscience.2009.05.060

Bardet, S. M., Ferran, J. L. E., Sanchez-Arrones, L., and Puelles, L. (2010). Ontogenetic expression of sonic hedgehog in the chicken subpallium. Front. Neuroanat. 4:28. doi: 10.3389/fnana.2010.00028

Behrens, J., von Kries, J. P., Kühl, M., Bruhn, L., Wedlich, D., Grosschedl, R., et al. (1996). Functional interaction of $\beta$-catenin with the transcription factor LEF-1. Nature 382, 638-642. doi: 10.1038/382638a0

Bielle, F., Griveau, A., Narboux-Nême, N., Vigneau, S., Sigrist, M., Arber, S., et al. (2005). Multiple origins of Cajal-Retzius cells at the borders of the developing pallium. Nat. Neurosci. 8, 1002-1012. doi: 10.1038/nn1511

Bininda-Emonds, O. R. P., and Hartmann, A.-M. (2017). "Mammalian evolution: the phylogenetics story," in Evolution of Nervous Systems, 2nd Edn, ed. embryo dissection/initial staging, and reviewed the first draft. GS designed part of the experiments, supervised part of the work, and reviewed the manuscript. MG conceptualized and designed the study with NS, supervised the study, wrote the final manuscript, and provided project administration and funding acquisition.

\section{FUNDING}

This work was funded by Democritus University of Thrace (Budget of the academic years 2017-2021).

\section{ACKNOWLEDGMENTS}

We acknowledge M. Batra, J. Grammenidou, and Z. Tzitzoudi, as well as J. Fysekis, J. Konstantelis, S. Kyroudis, and S. Polyzoes for their generous help. Furthermore, we would like to express our gratitude to $\mathrm{C}$. J. Charvet for sharing translational data regarding developmental events between mice and cats.

\section{SUPPLEMENTARY MATERIAL}

The Supplementary Material for this article can be found online at: https://www.frontiersin.org/articles/10.3389/fnana. 2021.785541/full\#supplementary-material

J. H. Kaas (Amsterdam: Elsevier), 77-86. doi: 10.1016/B978-0-12-804042-3.00 031-2

Bradbury, A. M., Cochran, J. N., McCurdy, V. J., Johnson, A. K., Brunson, B. L., Gray-Edwards, H., et al. (2013). Therapeutic response in feline Sandhoff disease despite immunity to intracranial gene therapy. Mol. Ther. 21, 1306-1315. doi: 10.1038/mt.2013.86

Brox, A., Puelles, L., Ferreiro, B., and Medina, L. (2004). Expression of the genes Emx1, Tbr1, and Eomes (Tbr2) in the telencephalon of Xenopus laevis confirms the existence of a ventral pallial division in all tetrapods. J. Comp. Neurol. 474, 562-577. doi: 10.1002/cne.20152

Buckley, R. M., Davis, B. W., Brashear, W. A., Farias, F. H. G., Kuroki, K., Graves, T., et al. (2020). A new domestic cat genome assembly based on long sequence reads empowers feline genomic medicine and identifies a novel gene for dwarfism. PLoS Genet. 16:e1008926. doi: 10.1371/journal.pgen.1008926

Bulfone, A., Martinez, S., Marigo, V., Campanella, M., Basile, A., Quaderi, N., et al. (1999). Expression pattern of the Tbr2 (Eomesodermin) gene during mouse and chick brain development. Mech. Dev. 84, 133-138. doi: 10.1016/S0925-4773(99) 00053-2

Bulfone, A., Smiga, S. M., Shimamura, K., Peterson, A., Puelles, L., and Rubenstein, J. L. R. (1995). T-Brain-1: a homolog of Brachyury whose expression defines molecularly distinct domains within the cerebral cortex. Neuron 15, 63-78. doi: 10.1016/0896-6273(95)90065-9

Bupesh, M., Abellán, A., and Medina, L. (2011). Genetic and experimental evidence supports the continuum of the central extended amygdala and a mutiple embryonic origin of its principal neurons. J. Comp. Neurol. 519, 3507-3531. doi: $10.1002 /$ cne. 22719

Butler, B. E., Hall, A. J., and Lomber, S. G. (2015). High-field functional imaging of pitch processing in auditory cortex of the cat. PLoS One 10:e0134362. doi: 10.1371/journal.pone.0134362

Carney, R. S. E., Cocas, L. A., Hirata, T., Mansfield, K., and Corbin, J. G. (2009). Differential regulation of telencephalic pallial-subpallial boundary patterning by Pax6 and Gsh2. Cereb. Cortex 19, 745-759. doi: 10.1093/cercor/bhn123 
Casarosa, S., Fode, C., and Guillemot, F. (1999). Mash1 regulates neurogenesis in the ventral telencephalon. Development 126, 525-534. doi: 10.1242/dev.126.3. 525

Catania, K. C. (2017). “The miniature neocortex of shrews-clues to mammalian brain evolution," in Evolution of Nervous Systems, ed. J. H. Kaas (Amsterdam: Elsevier), 181-186. doi: 10.1016/B978-0-12-804042-3.00038-5

Cave, J. W., Akiba, Y., Banerjee, K., Bhosle, S., Berlin, R., and Baker, H. (2010). Differential regulation of dopaminergic gene expression by Er81. J. Neurosci. 30, 4717-4724. doi: 10.1523/JNEUROSCI.0419-10.2010

Ceci, M. L., Pedraza, M., and de Carlos, J. A. (2012). The embryonic septum and ventral pallium, new sources of olfactory cortex cells. PLoS One 7:e44716. doi: 10.1371/journal.pone.0044716

Chou, S. J., and Tole, S. (2019). Lhx2, an evolutionarily conserved, multifunctional regulator of forebrain development. Brain Res. 1705, 1-14. doi: 10.1016/j. brainres.2018.02.046

Darch, H. T., Cerminara, N. L., Gilchrist, I. D., and Apps, R. (2020). Pre-movement changes in sensorimotor beta oscillations predict motor adaptation drive. Sci. Rep. 10:10167. doi: 10.1038/s41598-020-74833-z

Desfilis, E., Abellán, A., Sentandreu, V., and Medina, L. (2018). Expression of regulatory genes in the embryonic brain of a lizard and implications for understanding pallial organization and evolution. J. Comp. Neurol. 526, 166202. doi: 10.1002/cne.24329

Díaz-Guerra, E., Pignatelli, J., Nieto-Estévez, V., and Vicario-Abejón, C. (2013). Transcriptional regulation of olfactory bulb neurogenesis. Anat. Rec. 296, 1364-1382. doi: 10.1002/ar.22733

Døving, K. B., and Trotier, D. (1998). Structure and function of the vomeronasal organ. J. Exp. Biol. 201, 2913-2925. doi: 10.1242/jeb.201.21.2913

Duan, D., Fu, Y., Paxinos, G., and Watson, C. (2013). Spatiotemporal expression patterns of Pax6 in the brain of embryonic, newborn, and adult mice. Brain Struct. Funct. 218, 353-372. doi: 10.1007/s00429-012-0397-2

Eisenstat, D. D., Liu, J. K., Mione, M., Zhong, W., Yu, G., Anderson, S. A., et al. (1999). DLX-1, DLX-2, and DLX-5 expression define distinct stages of basal forebrain differentiation. J. Comp. Neurol. 414, 217-237. doi: 10.1002/(SICI) 1096-9861(19991115)414:2<217::AID-CNE6<3.0.CO;2-I

Englund, C., Fink, A., Lau, C., Pham, D., Daza, R. A. M., Bulfone, A., et al. (2005). Pax6, Tbr2, and Tbr1 are expressed sequentially by radial glia, intermediate progenitor cells, and postmitotic neurons in developing neocortex. J. Neurosci. 25, 247-251. doi: 10.1523/JNEUROSCI.2899-04.2005

Esclapez, M., Tillakaratne, N. J. K., Tobin, A. J., and Houser, C. R. (1993). Comparative localization of mRNAs encoding two forms of glutamic acid decarboxylase with nonradioactive in situ hybridization methods. J. Comp. Neurol. 331, 339-362. doi: 10.1002/cne.903310305

Esclapez, M., Tillakaratne, N., Kaufman, D., Tobin, A., and Houser, C. (1994). Comparative localization of two forms of glutamic acid decarboxylase and their mRNAs in rat brain supports the concept of functional differences between the forms. J. Neurosci. 14, 1834-1855. doi: 10.1523/JNEUROSCI.14-03-01834. 1994

Evans, H. E., and Sack, W. O. (1973). Prenatal development of domestic and laboratory mammals: growth curves, external features and selected references. Anat. Histol. Embryol. 2, 11-45. doi: 10.1111/j.1439-0264.1973.tb00253.x

Flames, N., Pla, R., Gelman, D. M., Rubenstein, J. L. R., Puelles, L., and Marín, O. (2007). Delineation of multiple subpallial progenitor domains by the combinatorial expression of transcriptional codes. J. Neurosci. 27, 9682-9695. doi: 10.1523/JNEUROSCI.2750-07.2007

Galceran, J., Miyashita-Lin, E. M., Devaney, E., Rubenstein, J. L. R., and Grosschedl, R. (2000). Hippocampus development and generation of dentate gyrus granule cells is regulated by LEF1. Development 127, 469-482. doi: 10.1242/dev.127.3. 469

Galuske, R. A. W., Munk, M. H. J., and Singer, W. (2019). Relation between gamma oscillations and neuronal plasticity in the visual cortex. Proc. Natl. Acad. Sci. U.S.A. 116, 23317-23325. doi: 10.1073/pnas.1901277116

Garcia-Calero, E., and Puelles, L. (2021). Development of the mouse anterior amygdalar radial unit marked by Lhx9-expression. Brain Struct. Funct. 226, 575-600. doi: 10.1007/s00429-020-02201-8

García-López, M., Abellán, A., Legaz, I., Rubenstein, J. L. R., Puelles, L., and Medina, L. (2008). Histogenetic compartments of the mouse centromedial and extended amygdala based on gene expression patterns during development. J. Comp. Neurol. 506, 46-74. doi: 10.1002/cne.21524
Gelman, D. M., Martini, F. J., Nóbrega-Pereira, S., Pierani, A., Kessaris, N., and Marín, O. (2009). The embryonic preoptic area is a novel source of cortical GABAergic interneurons. J. Neurosci. 29, 9380-9389. doi: 10.1523/ JNEUROSCI.0604-09.2009

Glatzle, M., Hoops, M., Kauffold, J., Seeger, J., and Fietz, S. A. (2017). Development of deep and upper neuronal layers in the domestic cat, sheep and pig neocortex. J. Vet. Med. Ser. C Anat. Histol. Embryol. 46, 397-404. doi: 10.1111/ahe.12282

Godbole, G., Roy, A., Shetty, A. S., and Tole, S. (2017). Novel functions of LHX2 and PAX6 in the developing telencephalon revealed upon combined loss of both genes. Neural Dev. 12, 1-8. doi: 10.1186/s13064-017-0097-y

Graff, E. C., Cochran, J. N., Kaelin, C. B., Day, K., Gray-Edwards, H. L., Watanabe, R., et al. (2020). PEA15 loss of function and defective cerebral development in the domestic cat. PLoS Genet. 16:e1008671. doi: 10.1371/journal.pgen.1008671

Gray-Edwards, H. L., Regier, D. S., Shirley, J. L., Randle, A. N., Salibi, N., Thomas, S. E., et al. (2017). Novel biomarkers of human GM1 gangliosidosis reflect the clinical efficacy of gene therapy in a feline model. Mol. Ther. 25, 892-903. doi: 10.1016/j.ymthe.2017.01.009

Guillemot, F. (2005). Cellular and molecular control of neurogenesis in the mammalian telencephalon. Curr. Opin. Cell Biol. 17, 639-647. doi: 10.1016/j. ceb.2005.09.006

Hevner, R. F., Neogi, T., Englund, C., Daza, R. A., and Fink, A. (2003). CajalRetzius cells in the mouse: transcription factors, neurotransmitters, and birthdays suggest a pallial origin. Dev. Brain Res. 141, 39-53. doi: 10.1016/ S0165-3806(02)00641-7

Hevner, R. F., Shi, L., Justice, N., Hsueh, Y. P., Sheng, M., Smiga, S., et al. (2001). Tbr1 regulates differentiation of the preplate and layer 6. Neuron 29, 353-366. doi: 10.1016/S0896-6273(01)00211-2

Hinds, J. W. (1968). Autoradiographic study of histogenesis in the mouse olfactory bulb. II. Cell proliferation and migration. J. Comp. Neurol. 134, 305-321. doi: 10.1002/cne.901340305

Hinova-Palova, D., Kotov, G., Landzhov, B., Edelstein, L., Iliev, A., Stanchev, S., et al. (2019). Cytoarchitecture of the dorsal claustrum of the cat: a quantitative Golgi study. J. Mol. Histol. 50, 435-457. doi: 10.1007/s10735-019-09839-7

Hodge, R. D., Garcia, A. J., Elsen, G. E., Nelson, B. R., Mussar, K. E., Reiner, S. L., et al. (2013). Tbr2 expression in Cajal-Retzius cells and intermediate neuronal progenitors is required for morphogenesis of the dentate gyrus. J. Neurosci. 33, 4165-4180. doi: 10.1523/JNEUROSCI.4185-12.2013

Imamura, F., and Greer, C. A. (2013). Pax6 regulates Tbr1 and Tbr2 expressions in olfactory bulb mitral cells. Mol. Cell. Neurosci. 54, 58-70. doi: 10.1016/j.mcn. 2013.01.002

Jacobowitz, D. M., and Abbott, L. C. (1997). Chemoarchitectonic Atlas of the Developing Mouse Brain. Boca Raton, FL: CRC Press. doi: 10.1201/ 9781466593411

Jasper, H. H., and Ajmone-Marsan, C. (1954). A Stereotaxic Atlas of the Diencephalon of the Cat. Ottawa, ON: The National Research Council of Canada. doi: 10.4224/21273363

Katarova, Z., Sekerková, G., Prodan, S., Mugnaini, E., and Szabó, G. (2000). Domain-restricted expression of two glutamic acid decarboxylase genes in midgestation mouse embryos. J. Comp. Neurol. 424, 607-627. doi: 10.1002/ 1096-9861(20000904)424:4<607::AID-CNE4<3.0.CO;2-C

Kelava, I., Lewitus, E., and Huttner, W. B. (2013). The secondary loss of gyrencephaly as an example of evolutionary phenotypical reversal. Front. Neuroanat. 7:16. doi: 10.3389/fnana.2013.00016

Kim, A. S., Anderson, S. A., Rubenstein, J. L., Lowenstein, D. H., and Pleasure, S. J. (2001). Pax-6 regulates expression of SFRP-2 and Wnt-7b in the developing CNS. J. Neurosci. 21, 3-7. doi: 10.1523/jneurosci.21-05-j0002.2001

Knospe, C. (2002). Periods and stages of the prenatal development of the domestic cat. Anat. Histol. Embryol. 31, 37-51. doi: 10.1046/j.1439-0264.2002.00360.x

Kocsis, K., Kiss, J., Csáki, A., and Halász, B. (2003). Location of putative glutamatergic neurons projecting to the medial preoptic area of the rat hypothalamus. Brain Res. Bull. 61, 459-468. doi: 10.1016/S0361-9230(03) 00180-1

Kremkow, J., Jin, J., Wang, Y., and Alonso, J. M. (2016). Principles underlying sensory map topography in primary visual cortex. Nature 533, 52-57. doi: 10.1038 /nature17936

Krettek, J. E., and Price, J. L. (1978b). Amygdaloid projections to subcortical structures within the basal forebrain and brainstem in the rat and cat. J. Comp. Neurol. 178, 225-253. doi: 10.1002/cne.901780204 
Krettek, J. E., and Price, J. L. (1978a). A description of the amygdaloid complex in the rat and cat with observations on intra-amygdaloid axonal connections. J. Comp. Neurol. 178, 255-279. doi: 10.1002/cne.901780205

Lavdas, A. A., Grigoriou, M., Pachnis, V., and Parnavelas, J. G. (1999). The medial ganglionic eminence gives rise to a population of early neurons in the developing cerebral cortex. J. Neurosci. 19, 7881-7888. doi: 10.1523/jneurosci. 19-18-07881.1999

Li, G., Kataoka, H., Coughlin, S. R., and Pleasure, S. J. (2009). Identification of a transient subpial neurogenic zone in the developing dentate gyrus and its regulation by Cxcl12 and reelin signaling. Development 136, 327-335. doi: $10.1242 /$ dev.025742

Liodis, P., Denaxa, M., Grigoriou, M., Akufo-Addo, C., Yanagawa, Y., and Pachnis, V. (2007). Lhx6 activity is required for the normal migration and specification of cortical interneuron subtypes. J. Neurosci. 27, 3078-3089. doi: 10.1523/ JNEUROSCI.3055-06.2007

Long, J. E., Garel, S., Alvarez-Dolado, M., Yoshikawa, K., Osumi, N., AlvarezBuylla, A., et al. (2007). Dlx-dependent and -independent regulation of olfactory bulb interneuron differentiation. J. Neurosci. 27, 3230-3243. doi: 10. 1523/JNEUROSCI.5265-06.2007

Luskin, M. B., and Shatz, C. J. (1985b). Studies of the earliest generated cells of the cat's visual cortex: cogeneration of subplate and marginal zones. J. Neurosci. 5, 1062-1075. doi: 10.1523/jneurosci.05-04-01062.1985

Luskin, M. B., and Shatz, C. J. (1985a). Neurogenesis of the cat's primary visual cortex. J. Comp. Neurol. 242, 611-631. doi: 10.1002/cne.902420409

Manger, P. R., Cort, J., Ebrahim, N., Goodman, A., Henning, J., Karolia, M., et al. (2008). Is 21st century neuroscience too focussed on the rat/mouse model of brain function and dysfunction? Front. Neuroanat. 2:5. doi: 10.3389/neuro.05. 005.2008

Markowitsch, H. J., and Pritzel, M. (1977). A stereotaxic atlas of the prefrontal cortex of the cat. Acta Neurobiol. Exp. 37, 63-81.

Martínez-Cerdeño, V., Cunningham, C. L., Camacho, J., Keiter, J. A., Ariza, J., Lovern, M., et al. (2016). Evolutionary origin of Tbr2-expressing precursor cells and the subventricular zone in the developing cortex. J. Comp. Neurol. 524, 433-447. doi: 10.1002/cne.23879

Martínez-Cerdeño, V., García-Moreno, F., Tosches, M. A., Csillag, A., Manger, P. R., and Molnár, Z. (2018). Update on forebrain evolution: from neurogenesis to thermogenesis. Semin. Cell Dev. Biol. 76, 15-22. doi: 10.1016/j.semcdb.2017. 09.034

Martynoga, B., Drechsel, D., and Guillemot, F. (2012). Molecular control of neurogenesis: a view from the mammalian cerebral cortex. Cold Spring Harb. Perspect. Biol. 4:a008359. doi: 10.1101/cshperspect.a008359

McCurdy, V. J., Rockwell, H. E., Arthur, J. R., Bradbury, A. M., Johnson, A. K., Randle, A. N., et al. (2015). Widespread correction of central nervous system disease after intracranial gene therapy in a feline model of Sandhoff disease. Gene Ther. 22, 181-189. doi: 10.1038/gt.2014.108

Medina, L. (2007). "Field homologies," in Evolution of Nervous Systems, ed. J. H. Kaas (Cambridge, MA: Academic Press), 73-87. doi: 10.1016/B0-12-370878-8/ 00097-5

Medina, L., Abellán, A., and Desfilis, E. (2019). Evolution of pallial areas and networks involved in sociality: comparison between mammals and sauropsids. Front. Physiol. 10:894. doi: 10.3389/fphys.2019.00894

Medina, L., Abellán, A., and Desfilis, E. (2021). Evolving views on the pallium. Brain Behav. Evol. 1-19. doi: 10.1159/000519260

Medina, L., and Abellán, A. (2012). "Subpallial structures," in The Mouse Nervous System, eds C. Watson, G. Paxinos, and L. Puelles (Cambridge, MA: Academic Press), 173-220. doi: 10.1016/B978-0-12-369497-3.10007-X

Medina, L., Brox, A., Legaz, I., García-López, M., and Puelles, L. (2005). Expression patterns of developmental regulatory genes show comparable divisions in the telencephalon of Xenopus and mouse: insights into the evolution of the forebrain. Brain Res. Bull. 66, 297-302. doi: 10.1016/j.brainresbull.2005.02.003

Medina, L., Legaz, I., González, G., De Castro, F., Rubenstein, J. L. R., and Puelles, L. (2004). Expression of Dbx1, neurogenin 2, semaphorin 5A, cadherin 8, and Emx1 distinguish ventral and lateral pallial histogenetic divisions in the developing mouse claustroamygdaloid complex. J. Comp. Neurol. 474, 504-523. doi: 10.1002/cne.20141

Molnár, Z., Métin, C., Stoykova, A., Tarabykin, V., Price, D. J., Francis, F., et al. (2006). Comparative aspects of cerebral cortical development. Eur. J. Neurosci. 23, 921-934. doi: 10.1111/j.1460-9568.2006.04611.x
Morales, L., Castro-Robles, B., Abellán, A., Desfilis, E., and Medina, L. (2021). A novel telencephalon-opto-hypothalamic morphogenetic domain coexpressing Foxg1 and Otp produces most of the glutamatergic neurons of the medial extended amygdala. J. Comp. Neurol. 529, 2418-2449. doi: 10.1002/cne.2 5103

Nóbrega-Pereira, S., Gelman, D., Bartolini, G., Pla, R., Pierani, A., and Marín, O. (2010). Origin and molecular specification of globus pallidus neurons. J. Neurosci. 30, 2824-2834. doi: 10.1523/JNEUROSCI.4023-09.2010

Ottem, E. N., Godwin, J. G., Krishnan, S., and Petersen, S. L. (2004). Dualphenotype GABA/glutamate neurons in adult preoptic area: sexual dimorphism and function. J. Neurosci. 24, 8097-8105. doi: 10.1523/JNEUROSCI.2267-04. 2004

Paxinos, G., and Ashwell, K. (2018). Atlas of the Developing Rat Nervous System, 4th Edn. Cambridge, MA: Academic Press.

Pontius, J. U., Mullikin, J. C., Smith, D. R., Lindblad-Toh, K., Gnerre, S., Clamp, M., et al. (2007). Initial sequence and comparative analysis of the cat genome. Genome Res. 17, 1675-1689. doi: 10.1101/gr.6380007

Puelles, L. (2014). "Development and evolution of the claustrum," in The Claustrum, eds J. R. Smythies, L. R. Edelstein, and V. S. Ramachandran (Cambridge, MA: Academic Press), 119-176. doi: 10.1016/B978-0-12-4045668.00004-0

Puelles, L. (2017). Comments on the updated tetrapartite pallium model in the mouse and chick, featuring a homologous claustro-insular complex. Brain Behav. Evol. 90, 171-189. doi: 10.1159/000479782

Puelles, L., Alonso, A., García-Calero, E., and Martínez-de-la-Torre, M. (2019). Concentric ring topology of mammalian cortical sectors and relevance for patterning studies. J. Comp. Neurol. 527, 1731-1752. doi: 10.1002/cne.24650

Puelles, L., and Ferran, J. L. E. (2012). Concept of neural genoarchitecture and its genomic fundament. Front. Neuroanat. 6:47. doi: 10.3389/fnana.2012.00047

Puelles, L., and Rubenstein, J. L. R. (2003). Forebrain gene expression domains and the evolving prosomeric model. Trends Neurosci. 26, 469-476. doi: 10.1016/ S0166-2236(03)00234-0

Puelles, L., and Rubenstein, J. L. R. (2015). A new scenario of hypothalamic organization: rationale of new hypotheses introduced in the updated prosomeric model. Front. Neuroanat. 9:27. doi: 10.3389/fnana.2015.00027

Puelles, L., Harrison, M., Paxinos, G., and Watson, C. (2013). A developmental ontology for the mammalian brain based on the prosomeric model. Trends Neurosci. 36, 570-578. doi: 10.1016/j.tins.2013.06.004

Puelles, L., Kuwana, E., Puelles, E., Bulfone, A., Shimamura, K., Keleher, J., et al. (2000). Pallial and subpallial derivatives in the embryonic chick and mouse telencephalon, traced by the expression of the genes Dlx-2, Emx-1, Nkx2.1, Pax-6, and Tbr-1. J. Comp. Neurol. 424, 409-438. doi: 10.1002/10969861(20000828)424:3<409::AID-CNE3<3.0.CO;2-7

Puelles, L., Ayad, A., Alonso, A., Sandoval, J. E., Martínez-de-la-Torre, M., Medina, L., et al. (2016a). Selective early expression of the orphan nuclear receptor Nr4a2 identifies the claustrum homolog in the avian mesopallium: Impact on sauropsidian/mammalian pallium comparisons. J. Comp. Neurol. 524, 665-703. doi: 10.1002/cne.23902

Puelles, L., Morales-Delgado, N., Merchán, P., Castro-Robles, B., Martínez-dela-Torre, M., Díaz, C., et al. (2016b). Radial and tangential migration of telencephalic somatostatin neurons originated from the mouse diagonal area. Brain Struct. Funct. 221, 3027-3065. doi: 10.1007/s00429-015-1086-8

Rakic, P. (2009). Evolution of the neocortex: a perspective from developmental biology. Nat. Rev. Neurosci. 10, 724-735. doi: 10.1038/nrn2719

Rash, B. G., Tomasi, S., Lim, H. D., Suh, C. Y., and Vaccarino, F. M. (2013). Cortical gyrification induced by fibroblast growth factor 2 in the mouse brain. J. Neurosci. 33, 10802-10814. doi: 10.1523/JNEUROSCI.3621-12.2013

Ruiz-Reig, N., Andres, B., Lamonerie, T., Theil, T., Fairén, A., and Studer, M. (2018). The caudo-ventral pallium is a novel pallial domain expressing Gdf10 and generating Ebf3-positive neurons of the medial amygdala. Brain Struct. Funct. 223, 3279-3295. doi: 10.1007/s00429-018-1687-0

Schambra, U. (2008). Prenatal Mouse Brain Atlas. Boston, MA: Springer US. doi: 10.1007/978-0-387-47093-1

Scott, P. P. (1977). The cat as an experimental animal. J. R. Soc. Med. 70, 1-3. doi: $10.1177 / 003591577707000102$

Sherk, H. (2014). "Physiology of the claustrum," in The Claustrum, eds J. R. Smythies, L. Edelstein, and V. S. Ramachandran (Amsterdam: Elsevier), 177191. doi: 10.1016/B978-0-12-404566-8.00005-2 
Shi, J., Wen, Z., Zhong, G., Yang, H., Wang, C., Huang, B., et al. (2020). Susceptibility of ferrets, cats, dogs, and other domesticated animals to SARScoronavirus 2. Science 368, 1016-1020. doi: 10.1126/science.abb7015

Shimamura, K., Hartigan, D. J., Martinez, S., Puelles, L., and Rubenstein, J. L. R. (1995). Longitudinal organization of the anterior neural plate and neural tube. Development 121, 3923-3933. doi: 10.1242/dev.121.12.3923

Smith-Fernandez, A., Pieau, C., Repérant, J., Boncinelli, E., and Wassef, M. (1998). Expression of the Emx-1 and Dlx-1 homeobox genes define three molecularly distinct domains in the telencephalon of mouse, chick, turtle and frog embryos: implications for the evolution of telencephalic subdivisions in amniotes. Development 125, 2099-2111. doi: 10.1242/dev.125.11. 2099

Stenman, J., Yu, R. T., Evans, R. M., and Campbell, K. (2003b). Tlx and Pax6 cooperate genetically to establish the pallio-subpallial boundary in the embryonic mouse telencephalon. Development 130, 1113-1122. doi: 10.1242/dev.00328

Stenman, J., Toresson, H., and Campbell, K. (2003a). Identification of two distinct progenitor populations in the lateral ganglionic eminence: implications for striatal and olfactory bulb neurogenesis. J. Neurosci. 23, 167-174. doi: 10.1523/ jneurosci.23-01-00167.2003

Stolzberg, D., Wong, C., Butler, B. E., and Lomber, S. G. (2017). Catlas: an magnetic resonance imaging-based three-dimensional cortical atlas and tissue probability maps for the domestic cat (Felis catus). J. Comp. Neurol. 525, 3190-3206. doi: $10.1002 /$ cne. 24271

Striedter, G. F. (2007). "A history of ideas in evolutionary neuroscience," in Evolution of Nervous Systems, ed. J. H. Kaas (Amsterdam: Elsevier), 1-15. doi: 10.1016/B0-12-370878-8/00096-3

Stylianopoulou, E., Kalamakis, G., Pitsiani, M., Fysekis, I., Ypsilantis, P., Simopoulos, C., et al. (2016). HSPC280, a winged helix protein expressed in the subventricular zone of the developing ganglionic eminences, inhibits neuronal differentiation. Histochem. Cell Biol. 145, 175-184. doi: 10.1007/s00418-0151380-9

Sugiyama, T., Osumi, N., and Katsuyama, Y. (2013). The germinal matrices in the developing dentate gyrus are composed of neuronal progenitors at distinct differentiation stages. Dev. Dyn. 242, 1442-1453. doi: 10.1002/dvdy.24035

Sussel, L., Marin, O., Kimura, S., and Rubenstein, J. L. R. (1999). Loss of Nkx2.1 homeobox gene function results in a ventral to dorsal molecular respecification within the basal telencephalon: evidence for a transformation of the pallidum into the striatum. Development 126, 3359-3370. doi: 10.1242/dev.126.15.3359

Tarozzo, G., Peretto, P., and Fasolo, A. (1995). Cell migration from the olfactory placode and the ontogeny of the neuroenedocrine compartments. Zool. Sci. 12, 367-383. doi: 10.2108/zsj.12.367

Tissir, F., Ravni, A., Achouri, Y., Riethmacher, D., Meyer, G., and Goffinet, A. M. (2009). DeltaNp73 regulates neuronal survival in vivo. Proc. Natl. Acad. Sci. U.S.A. 106, 16871-16876. doi: 10.1073/pnas.0903191106

Touzot, A., Ruiz-Reig, N., Vitalis, T., and Studer, M. (2016). Molecular control of two novel migratory paths for CGE-derived interneurons in the developing mouse brain. Dev. 143, 1753-1765. doi: 10.1242/dev.131102

Tuoc, T. C., and Stoykova, A. (2008). Er81 is a downstream target of Pax6 in cortical progenitors. BMC Dev. Biol. 8:23. doi: 10.1186/1471-213X-8-23
Urbán, N., and Guillemot, F. (2014). Neurogenesis in the embryonic and adult brain: same regulators, different roles. Front. Cell. Neurosci. 8:396. doi: 10.3389/ fncel.2014.00396

Valdés-Cruz, A., Villasana-Salazar, B., Williams, B., Martínez-Vargas, D., Magdaleno-Madrigal, V. M., Almazán-Alvarado, S., et al. (2019). Transcranial focal electrical stimulation via concentric ring electrodes in freely moving cats: antiepileptogenic and postictal effects. Exp. Neurol. 320:113012. doi: 10.1016/j. expneurol.2019.113012

Watson, C., and Puelles, L. (2017). Developmental gene expression in the mouse clarifies the organization of the claustrum and related endopiriform nuclei. J. Comp. Neurol. 525, 1499-1508. doi: 10.1002/cne.24034

Watson, C., Mitchelle, A., and Puelles, L. (2017). "A new mammalian brain ontology based on developmental gene expression," in Evolution of Nervous Systems, ed. J. H. Kaas (Amsterdam: Elsevier), 53-75. doi: 10.1016/B978-0-12804042-3.00030-0

Winpenny, E., Lebel-Potter, M., Fernandez, M. E., Brill, M. S., Götz, M., Guillemot, F., et al. (2011). Sequential generation of olfactory bulb glutamatergic neurons by Neurog2-expressing precursor cells. Neural Dev. 6, 1-18. doi: 10.1186/17498104-6-12

Workman, A. D., Charvet, C. J., Clancy, B., Darlington, R. B., and Finlay, B. L. (2013). Modeling transformations of neurodevelopmental sequences across mammalian species. J. Neurosci. 33, 7368-7383. doi: 10.1523/JNEUROSCI. 5746-12.2013

Yoshida, M., Assimacopoulos, S., Jones, K. R., and Grove, E. A. (2006). Massive loss of Cajal-Retzius cells does not disrupt neocortical layer order. Development 133, 537-545. doi: 10.1242/dev.02209

Yun, K., Potter, S., and Rubenstein, J. L. R. (2001). Gsh2 and Pax6 play complementary roles in dorsoventral patterning of the mammalian telencephalon. Development 128, 193-205. doi: 10.1242/dev.128.2.193

Zilles, K., Palomero-Gallagher, N., and Amunts, K. (2013). Development of cortical folding during evolution and ontogeny. Trends Neurosci. 36, 275-284. doi: 10.1016/j.tins.2013.01.006

Conflict of Interest: The authors declare that the research was conducted in the absence of any commercial or financial relationships that could be construed as a potential conflict of interest.

Publisher's Note: All claims expressed in this article are solely those of the authors and do not necessarily represent those of their affiliated organizations, or those of the publisher, the editors and the reviewers. Any product that may be evaluated in this article, or claim that may be made by its manufacturer, is not guaranteed or endorsed by the publisher.

Copyright (c) 2021 Siskos, Ververidis, Skavdis and Grigoriou. This is an open-access article distributed under the terms of the Creative Commons Attribution License (CC BY). The use, distribution or reproduction in other forums is permitted, provided the original author(s) and the copyright owner(s) are credited and that the original publication in this journal is cited, in accordance with accepted academic practice. No use, distribution or reproduction is permitted which does not comply with these terms. 